\title{
A Fast Monte Carlo Simulation for the International Linear Collider Detector
}

\author{
Daniel Furse \\ Office of Science, SULI Program \\ Georgia Institue of Technology \\ Stanford Linear Accelerator Center \\ Menlo Park, California
}

August, 2005

Prepared in partial fulfillment of the requirements of the Office of Science, U.S. Department of Energy Science Undergraduate Laboratory Internship (SULI) Program under the direction of Dr. Timothy Barklow in the Particle Physics Division of Stanford Linear Accelerator Center.

Participant:

Signature

Research Advisor:

Signature 


\title{
A Fast Monte Carlo Simulation for the International Linear Collider Detector
}

\author{
Daniel Furse
}

3 Aug 2005

\begin{abstract}
The following paper contains details concerning the motivation for, implementation and performance of a Java-based fast Monte Carlo simulation for a detector designed to be used in the International Linear Collider. This simulation, presently included in the SLAC ILC group's org.lcsim package, reads in standard model or SUSY events in STDHEP file format, stochastically simulates the blurring in physics measurements caused by intrinsic detector error, and writes out an LCIO format file containing a set of final particles statistically similar to those that would have found by a full Monte Carlo simulation. In addition to the reconstructed particles themselves, descriptions of the calorimeter hit clusters and tracks that these particles would have produced are also included in the LCIO output. These output files can then be put through various analysis codes in order to characterize the effectiveness of a hypothetical detector at extracting relevant physical information about an event. Such a tool is extremely useful in preliminary detector research and development, as full simulations are extremely cumbersome and taxing on processor resources; a fast, efficient Monte Carlo can facilitate and even make possible detector physics studies that would be very impractical with the full simulation
\end{abstract}


by sacrificing what is in many cases inappropriate attention to detail for valuable gains in time required for results. 


\section{Background}

The International Linear Collider collaboration was officially formed in 1998 following a meeting of the group's inaugural committee at ICHEP in Vancouver and has persisted ever since at the forefront of the worldwide effort toward the eventual construction of a $\mathrm{TeV}$ range $e^{+} e^{-}$linear collider. This group is secondarily split along continental lines into three main semi-autonomous collaborations. In Europe and centered at Hamburg (part of the DESY group) is the ECFA collaboration, which did a great deal of work early on in designing a mid-size, $B=4 T$ detector; their main design report [1] continues to be a good source of ballpark figures and parameterizations for detector performance. In Asia and centered in Japan at KEK is the ACFA collaboration, which mainly works on a lower B-field, larger tracker detector design. Finally, strongly represented at SLAC and with groups scattered over most of North America is the ALCPG group, with whom this project has been conducted.

Clearly, most of the attention given to plans for this future accelerator falls on the design of the detector itself, which is natural given its proportionately higher cost and complexity. Since the energy ranges in which this collider will be operating demand a linear design, this reduces the number of possible detector sites to one (compare, say, CERN with four different detectors), which in turn forces a number of compromises to be drawn. The detector that is finally built must have good position and energy resolution in all of its component parts (rather than, say, using different detectors for different types of measurements) and be nearly hermetically sealed in order to ensure an accurate characterization of collision events. This all must be done while optimizing cost: a task which demands high-quality, easily accessible knowledge of what an arbitrary detector design is capable of. It is the pursuit of this sort of performance data that drives almost all the computer simulation efforts for the ILC here at SLAC. 


\section{Motivation}

The task of fully simulating the response of a particle detector to a high energy physics event is, without a doubt, computationally daunting; so much so in fact that it must be handled as a set of separate problems. The entire task is quite naturally broken down into three phases, each with an independent goal and different file type, allowing for analysis code to be conveniently run at points in the middle of the full process. The first of these phases is event generation, whereby a physicist can produce a file containing potentially millions of different instances of a particular high-energy event. Event generation programs offer varying degrees of control over the type and realisticness of events produced, allowing a user to condition the output according to his needs. This flexibility is extremely important in producing meaningful statistical studies of detector behavior, and we exploit it fully in conducting the studies in the subsequent sections on performance. The particular event generation program used here is called Whizard, which uses Monte Carlo methods to simulate many details of particle events, including decay chains, bremsstrahlung, and gluon radiation. Whizard will typically take electrons and positrons and produce a specific type of event, which it then naturally simulates on through decays down to particles stable enough to interact with a normal particle detector; this list of particles includes photons, electrons,

muons, neutrinos, pions, $K_{\text {long }}^{0}$-s, protons and neutrons. All of this event information (particle types, momenta, energies, parentage information, etc.) is written to an output file in STDHEP format, which is the input file for the next stage.

The next step is to take the final state particles and simulate the minute details of their interaction with the detector. The program we typically use for this is a variant of the widely-known Geant4 program made at CERN for their simulation studies; its installation at SLAC is called SLIC. SLIC will take an STDHEP file and detector description (usually formatted as Java code) as input, producing raw detector hits as information in the output file. 
These hits are really just singlets or doublets of data, being either only an id flag identifying which part of the detector was activated (tracker elements produce such hits) or and id flag and a value (such as a calorimeter cell would produce). In order to produce such a file, Geant4 takes the final state particles and swims them through the simulated detector, allowing photons to randomly pair-produce as they pass detector components, allowing neutrons to produce showers of hits in the calorimeter, among other things, all in a very realistic and exhaustive way, particle by particle.

The final step in a full simulation is reconstruction; this section is different than the other two in that the algorithms implemented are also used in reconstructing events that produced actual detector data. The goal of the reconstruction programming is to take the raw data in the LCIO file produced by SLIC and reproduce the STDHEP events as closely as possible, thus giving a good measure of how well detector and code are working together to extract physics from data. To be most accurate, the reconstruction code is really part of the detector itself, as weak points in the algorithms are manifestly indistinguishable from errors intrinsic to the detector - it is therefore of utmost importance to have the best possible reconstruction code with little regard for speed. Currently, the ILC group at SLAC is still fine-tuning the calorimeter cluster reconstruction portions of their code, which gives a sense of the seriousness of this step.

Clearly, this is a long and complicated process (a flowchart of the data path can be found in the appendix) where accuracy is absolutely the first priority, a fact which meets many needs very well. But there is also a large and important set of information concerning general detector properties and dependances of physics data quality on said properties that elicits a much different priority ordering. When trying to get a good range for the solenoidal field needed in the detector or when trying to estimate what the best size is for a tracking chamber in a detector looking for certain Higgs events, a tool like the full SLIC + reconstruction is far from ideal. It is this type of data 
and the need for it that is best addressed by a fast Monte Carlo simulation, one that statistically matches the full Monte Carlo as closely as possible in important areas without getting into the details of raw data. A good Monte Carlo simulator will be readily reconfigured to simulate different detector descriptions, all while remaining fast and satisfactorily faithful to the full simulation. Bringing to fruition such a simulator and optimizing it has been the primary task of this project.

\section{Implementation}

The group at the spearhead of SLAC's ILC-related simulation efforts is currently developing a Java-based reconstruction and analysis package called org.lcsim, which can be run either stand-alone or plugged into the Java Analysis Studio (JAS) 3 framework. From JAS3, one can load and run analyses on almost any section of the full org.lcsim package of classes and generate histograms and fitted curves for any set of data. The fast Monte Carlo simulator will be a part of this package, and itself can be run either stand-alone or inside of JAS3. Also presently included in the org.lcsim package is a snippet of analysis code written to evaluate the performance of the fast MC. This code divides the detector into thrust hemispheres using the plane normal to the thrust axis calculated for particles created in the detector and then generates histograms for the jet energy resolution by calling all the particles in each thrust hemisphere members of a jet. Histograms generated by this code for two-jet $e^{+} e^{-} \rightarrow u \bar{u}$ events can be found in the performance section of the paper.

The fast MC itself, being an alternative to SLIC + reconstruction, needs to take the type of input SLIC works with and generate files similar to those the reconstruction algorithm generates. Hence, the fast $\mathrm{MC}$ will read in STDHEP files, then directly smear each final state particle and create a detector object appropriate for the particle type and charge, along with a 
reconstructed particle object for all particles except neutrinos. This means that different sets of smearing algorithms are needed depending on the part of the detector a specific particle interacts with, the different detector component behaviors being governed by independent sets of parameters. A good initial source for developing effective methods has again been provided by the DESY group's TESLA design report[1]. This report provides some excellent information as to the dependences of detector component resolutions on event variables as well as the dependences of these resolutions on construction variables, which while not directly accessible in the fast MC do provide realistic limits on the MC parameter ranges. Another good source of implementation examples is an older fast MC program written in FORTRAN called SIMDET[2]; we borrow lightly from this program and actually have used its performance characteristics as a baseline goal which we hoped to surpass. All of the different methods we implemented are outlined in the sections below, which break down the mathematical core of the program by detector components.

\subsection{Calorimeter Methods}

For all of the dynamical variables measurable from a cluster of calorimeter hits associated with a particle (energy, momentum direction and particle species, to a degree), we take the STDHEP particle's properties and smear them according to various stochastic formulae:

- Energy Smearing:

$$
E_{\text {recon }}=E+\mathcal{R}_{g} \cdot\left\{E\left(\frac{a}{\sqrt{E}}+b\right)\right\}
$$

Where $\mathcal{R}_{g}$ is a gaussian random number with a standard deviation of one and a mean of zero, and $E$ is the energy of the STDHEP file 
particle for which we're generating a cluster. There are separate parameters $a$ and $b$ for both the electromagnetic calorimeter and the hadronic calorimeter.

- Position (Momentum) Smearing:

$$
\vec{r}_{\text {recon }}=r_{\text {det }}(\theta, \phi) \cdot\left\{\frac{a}{\sqrt{a^{2}+b^{2} \mathcal{R}_{g}^{2}}} \hat{r}+\frac{b \mathcal{R}_{g}}{\sqrt{a^{2}+b^{2} \mathcal{R}_{g}^{2}}}\left(\sin (\mathcal{R} \phi) \hat{v}_{1}+\cos (\mathcal{R} \phi) \hat{v}_{2}\right)\right\}
$$

Where again $\mathcal{R}_{g}$ is a gaussian random number, plain $\mathcal{R}$ is a random number flatly distributed between zero and one, $\hat{r}$ is a unit vector pointing from the interaction point to the STDHEP particle's actual position at the face of the calorimeter, $\theta$ is the angle $\hat{r}$ makes with the beampipe, $\phi$ is the azimuthal angle of $\hat{r}, \hat{v}_{1,2}$ are any unit vectors that along with $\hat{r}$ comprise an orthonormal set, and finally where the function $r_{\text {det }}(\theta, \phi)$ describes the shape of the inside of the calorimeter.

- Efficiency:

$$
P(E)=1-\frac{1}{1+e^{b \cdot(E-a)}}
$$

This equation gives the probability of a particle of energy $\mathrm{E}$ to be observed in a calorimeter. The parameters $a$ and $b$ are called, respectively, the onset and the sharpness. It may be immediately clear, but $a$ is the energy at which a particle will be observed half of the time, and the parameter $b$ is $\left.2 \cdot \frac{d P}{d E}\right|_{E=a}$. 
This function may be made arbitrarily close to step-like as the user wants simply by increasing b to a very high value, normally 10,000. A picture of the function can be found below, in Figure 1:

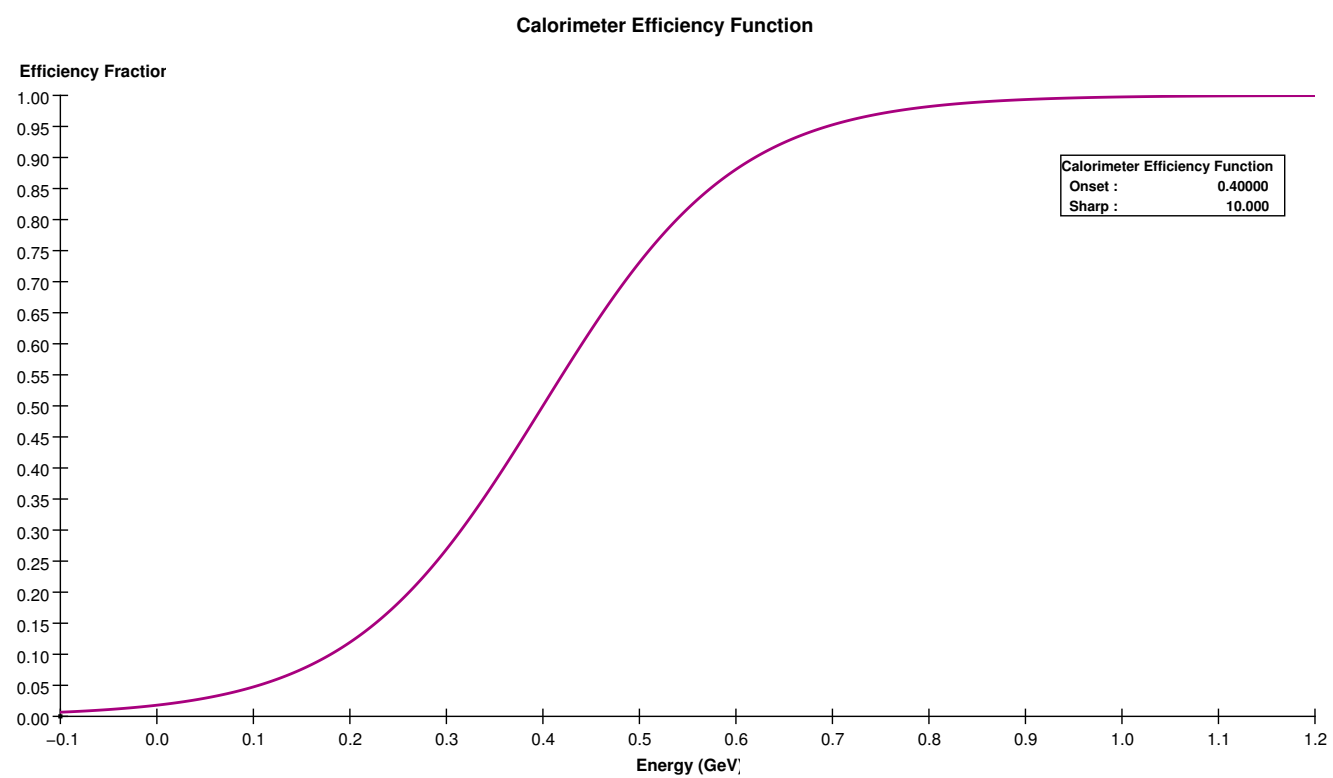

Figure 1: The basic efficiency function used in the org.lcsim fast MC; this function is incidentally called the Woods-Saxon potential and is used to model the strong-force binding potential nucleons feel inside heavy nuclei.

This concludes the list of methods that are easily associated with a single formula. The following sections concern smearing methods that are more programmatic; incidentally, these phenomena affect the physical results the detector can deliver to a much lesser degree than those described above.

- Particle Identfification:

The ability of the detector to correctly identify particles is modeled according to the following prescription. All stable, charged tracks 
are at first assumed to be pions (this is, statistically, an excellent assumption), but if the particle actually is not a pion the program will throw a flatly distributed random number to determine whether or not the particle is correctly identified, with separate efficiencies for protons, electrons, and muons. If the random number is below the efficiency for the particle specified, the fast MC identifies the non-pion particle correctly in the output file. Similarly, all neutral clusters are assumed to be associated with $K_{l o n g}^{0}$ impacts, but there is an efficiency for correct neutron identification. Photons leave such a distinctive burst in the electromagnetic calorimeter that they are assumed to be correctly identified $100 \%$ of the time, which is again an excellent assumption. Currently, all identification efficiencies are constant throughout the energy range of the detector, due to the fact that the actual efficiencies and dependances we are trying to mimic are completely dependent on the quality of the reconstruction algorithms which, as mentioned previously, are still being developed. As soon as we can assess the function that best matches the identification quality's dependence, it can be easily included in the program.

\section{- Cluster Overlap Compensation}

While the effects of having clusters overlap are quite small (by design) in the current ILC detector, the method is so mathematically interesting that it has been included anyway. There are, fundamentally, two separate phenomena that can occur as a result of clusters landing on top of each other. The more severe of these two processes is complete cluster confusion, in which two clusters of hits are in such close proximity and so entangled with each other that a reconstruction program will more than likely confuse them as one large cluster. This effect is simple to model, with the probability for any two clusters $i$ and $j$ being confused 
given by:

$$
P_{i j}=\iint_{C a l} \mathrm{~d}^{2} r\left(\frac{1}{\sqrt{2 \pi b_{i}^{2}}} e^{\frac{\left(\vec{r}_{i}-\vec{r}\right)^{2}}{4 b_{i}^{2}}}\right) \cdot\left(\frac{1}{\sqrt{2 \pi b_{j}^{2}}} e^{\frac{\left(\vec{r}_{j}-\vec{r}\right)^{2}}{4 b_{j}^{2}}}\right)
$$

Where the integral is over the surface of the calorimeter face, and the parameter $b_{i}$ could potentially depend on particle $i$ 's energy and type. In a current version (still in testing), all $b_{i}$ are constant, and set to the same value as the $b$ mentioned parameter in the momentum smearing section. Since the gaussians are symmetrical, the probability of confusion only depends on the separation of the centroids; the formula resulting from evaluating the integral above is how the dependence is actually implemented in the program:

$$
P_{i j}=e^{-\frac{\left\|\vec{r}_{i}-\vec{r}_{j}\right\|^{2}}{8 b^{2}}}
$$

To actually simulate confusing clusters and make use of the probability formula stated above, the program makes a list of integers with as many elements as there are clusters, and then runs through all possible combinations of clusters. If a flat random number compared with $P_{i j}$ as calculated above indicates that a certain combination $i j$ should be confused, the program will put the value of the lower of the two indices $i$ and $j$ into the slot in the list corresponding to the higher index, then proceed to the next combination; proceeding in this manner causes trees of confused hits to branch outward toward higher numbers, with the base node of the tree having the lowest index of all the cluseters it contains. After this process is complete, the program runs backwards over the list containing the confusion information conveniently enabling the program to run over any trees of hits it has created from branch to root, since a lower-numbered node will be closer to the 
root of a given tree. While scanning, if bin $i$ contains a value $j$ different from -1 , the program reassigns the position of cluster $j$ as an energy-weighted average of $i$ 's position and $j$ ?s position, dumps $i$ ? s energy into cluster $j$, and then removes cluster $i$ from the list. The list containing the confusion data is then dumped, and the remaining list of clusters represents the list of clusters that the reconstruction program actually has a chance of generating.

The other aspect of cluster confusion is the difficulty in dividing energy up between clusters that lie in close proximity. In order to simulate this effect accurately, we will remind ourselves of the fact that if a group of clusters is reasonably isolated, one can very accurately know the total energy of the cluster while still making significant errors in assigning partial energies to the individual clusters in the group. We can take such a group of isolated clusters and let it include the entire calorimeter, and then we arrive at the fact that the total energy deposited in the calorimeter should be an invariant quantity under any reasonable cluster confusion simulation. When we look (quite suggestively) at cluster confusions as transformations acting on the configuration of hits in the detector that must preserve a certain kind of sum, we see that energy partitioning errors for $n$ calorimeter clusters can be very well modeled by transformations belonging to the group $S O(n)$. This is done by introducing a stochastic $S O(n)$ smearing operator which acts on an energy vector defined as having elements $\sqrt{E_{i}}$, where $E_{i}$ is the energy of the $i$ th cluster; hence $\overrightarrow{\sqrt{E_{i}}} \cdot \overrightarrow{\overrightarrow{E_{i}}}=\sum_{i} E_{i}$ is preserved. Since we demand that the smearing operator be part of $S O(n)$, we know it will have the following form:

$$
\mathcal{O}=e^{c_{i j} g_{i j}}
$$


where $g_{i j}$ are the $\frac{n(n+1)}{2}$ generators of $S O(n)$ and the coefficients $c_{i j}$ are free for us to define as we choose. Since $c_{i j}$ represents the angle through which we want to rotate or smear the energy between clusters $i$ and $j$, we will want to restrict $c_{i j}$ to be on the interval $\left[-\frac{\pi}{2}, \frac{\pi}{2}\right]$, since the functions $\cos ^{2} \theta$ and $\sin ^{2} \theta$ are bijective on $\left[0, \frac{\pi}{2}\right]$ and we want to allow the vector to smear symmetrically in both directions without double counting any particular configuration. Hence, we choose our angle to be:

$$
c_{i j}=P_{i j} \mathcal{R}_{g}-\frac{\pi}{2} \cdot\left\lfloor\frac{P_{i j} \mathcal{R}_{g}}{\pi / 2}\right\rfloor
$$

Where the mean $P_{i j}$ of the gaussian portion is calculated in exactly the same way as the probability for completely confusing clusters (see Equation 4), except the standard deviations of the gaussians $\left(b_{i j}\right)$ may be different in this case. Using such a definition for $c_{i j}$ causes the probability of smearing to an angle $\theta$ to be given by:

$$
P(\theta)=\frac{1}{\sqrt{2 \pi P_{i j}^{2}}} \sum_{n=0}^{\infty} e^{-\frac{\left(\theta+n \frac{\pi}{4}\right)^{2}}{2 P_{i j}^{2}}}=\frac{e^{-\frac{\theta^{2}}{2 P_{i j}^{2}}}}{\sqrt{2 \pi P_{i j}^{2}}} \sum_{n=0}^{\infty} e^{-\frac{n^{2} \pi^{2}}{36 P_{i j}^{2}}}
$$

The second infinite series on the line is certainly convergent (by the integral test, if you like), and can be readily calculated exploiting the fact that $P(\theta)$ is normalized over $\left[-\frac{\pi}{2}, \frac{\pi}{2}\right]$, which is itself easily demonstrated by exploiting the linearity of integrals:

$$
\sum_{n=0}^{\infty} e^{-} \frac{n^{2} \pi^{2}}{36 P_{i j}^{2}}=\operatorname{erf}\left(\frac{\pi / 2}{\sqrt{2 P_{i j}^{2}}}\right)^{-1}
$$

which yields an expression for $P(\theta)$ that has no series in it: 


$$
P(\theta)=\frac{1}{\sqrt{2 \pi P_{i j}^{2}}} \cdot \operatorname{erf}\left(\frac{\pi / 2}{\sqrt{2 P_{i j}^{2}}}\right)^{-1} e^{-\frac{\theta^{2}}{2 P_{i j}^{2}}}
$$

This concludes our exhaustive treatment of the important feature of cluster confusion; an implementation of the methods described here might be made available in a future version of org.lcsim when the opportunity for tailoring the parameters to match the finished reconstruction algorithm exists.

\subsection{Tracker Methods}

The simulation of tracker error is much more modular and direct than the simulation of calorimeter error; indeed, there is one process performed on each track which simultaneously and interdependantly smears all the physical variables we are concerned with. The procedure requires a track to be parameterized as a five element vector, acted upon by a stochastic operator called the covariant error matrix. Complete details of these methods and a simple variation on them are included below.

\section{- Conversion to DOCA Parameters}

The acronym DOCA is an abbreviation for "distance of closest approach", and the DOCA parameters are a set of five variables that specify a helical trajectory winding around the detector's magnetic field by describing it at the point of closest approach to the beam axis. The DOCA parameters $x_{i}$ are defined as follows (references to a cartesian system assume that the $\mathrm{z}$ axis is along the detector axis):

$x_{0}=d_{0}$ is the helix's distance of closest approach to the z-axis.

$-x_{1}=\phi_{0}$ is the angle the projection of the track in the $\mathrm{x}-\mathrm{y}$ plane makes with the $\mathrm{x}$-axis at the point of closest approach 
to the $\mathrm{z}$ axis.

$-x_{2}=\Omega$ is the signed curvature of the track, and is directly proportional to the momentum of the track in the $\mathrm{x}-\mathrm{y}$ plane, which is itelf often called the transverse momentum.

$-x_{3}=z_{0}$ is the $\mathrm{z}$ coordinate of the track at the point of closest approach.

$-x_{4}=\tan \lambda$ where $\lambda$ is the angle the track makes with the $\mathrm{x}-\mathrm{y}$ plane at the point of closest approach.

These five variables are exactly enough to specify the position and momentum of the particle track, since the fact that these are defined to specify the closest approach position on the track implies the constraint $\vec{p}_{t} \cdot \vec{r}=0$, thus eliminating one of the coordinates from the $\vec{x}, \vec{p}$ set for the particle. The DOCA parameters are especially convenient for calculating the large set of error matrices discussed in the following section, and the DOCA variables are the ones we'll be working in unless otherwise noted.

- Covariant Track Smearing

The DOCA variables are quite natural for the detector, but they are interdependent; a small error in the curvature will certainly result in an erroneous measurement of, say, $\tan \lambda$, so it?s necessary to treat this aspect of the problem in a valid statistical fashion. The correct formalism is found by calculating a real, symmetric error matrix (which is also known as the covariance matrix) for the detector which describes how errors couple between variables. Given an error matrix, we can get the statistically independent variables by finding the eigenvectors $\vec{v}_{i}$ (which correspond to these independent combinations), whose distributions will be governed by eigenvalues $\sigma_{i}$. We then smear the track according to the matrix by using the following procedure: 


$$
\vec{X}_{\text {recon }}=\vec{X}+\left(\begin{array}{cccc}
\mid & \mid & \cdots & \mid \\
\vec{v}_{1} & \vec{v}_{2} & \cdots & \vec{v}_{5} \\
\mid & \mid & \cdots & \mid
\end{array}\right)\left(\begin{array}{c}
\mathcal{R}_{g 1} \cdot \sigma_{1} \\
\mathcal{R}_{g 2} \cdot \sigma_{2} \\
\vdots \\
\mathcal{R}_{g 5} \cdot \sigma_{5}
\end{array}\right)
$$

Where the set $\mathcal{R}_{g i}$ is a group of 5 independently drawn gaussian random numbers and recall that the set $\sigma_{i}$ are the square roots of the eigenvalues. Hence, the components of momentum and position are all simultaneously taken care of. The main drawback of this method, complete and elegant as it is, is the difficulty of calculating the covariance matrices and implementing them. Since the tracker itself is made up of a set of concentric cylinders, the error matrices describing the tracker will change depending on if the track passes through the endcaps or the barrels of the set. Also, there is an energy dependence on the resolution of the tracker for the various physical variables, and since the detector is not spherically symmetric there will also be a dependence in the matrix on the $\tan \lambda$ variable. These dependences are taken into account not by constructing an appropriate matrix-valued function, but rather by calculating constant matrices on a discrete lattice of points $(E, \lambda)$ and linearly interpolating matrices as they are needed. Again, we are faced with the problem that changing the description of the detector is quite difficult, and in order for the fast MC to be an effective tool for the types of physics studies it was designed to this cumbersomeness can be a serious drawback. Answering this shortcoming is a method for quickly modifying the distributions the matrices produce without changing the way the errors propagate between variables, the details of which are described in the following section.

- Modified Covariant Track Smearing 
Often, the object of a certain physics study of a detector is to determine the dependence of, say, the average mass resolution for a particle on an intrinsic detector resolution. With the present fast $\mathrm{MC}$ this is very easy to do with calorimeter resolutions since the user has direct access to these resolutions, but what about studies involving dependences on tracker resolutions? This is much more difficult to do since the matrix smears all the variables simultaneously. However, it should be mathematically possible to modify the matrices in such a way as to impose a resolution on a certain variable while leaving the error mixing properties unaffected. This is indeed true, though figuring out the method is somewhat involved. First of all, we must analyze and interpret the covariance matrix itself: how does the probability of adding a small error DOCA vector $\delta \vec{X}$ to the original DOCA parameter vector $\vec{X}$ depend on the covariance matrix $\hat{C}$ ? In order to answer this question, we must take another look at the method described in the previous section. Firstly, we can rewrite Equation 11 in the following way:

$$
\vec{X}_{\text {recon }}=\vec{X}+\sum_{k=1}^{5} \mathcal{R}_{g k} \sigma_{k} \vec{v}_{k}
$$

Upon inspection we see first of all that the set of vectors $\vec{v}_{k}$ form an orthonormal basis, being the eigenvectors of a hermitian matrix; as such they differ from the standard Cartesian set only by a transformation belonging to $S O(5)$. Also, we see that the standard deviation of the distribution describing the probability of drawing a certain component in the direction $\vec{v}_{k}$ is exactly $\sigma_{k}$. We can assimilate this information in a single statement by writing down the probability of drawing a correction vector $\delta \vec{X}$ : 


$$
P(\delta \vec{X})=\prod_{k=1}^{5} \frac{1}{\sqrt{2 \pi \sigma_{k}}} \cdot e^{-\sum_{k=1}^{5} \frac{\left(\delta \vec{X} \cdot \vec{v}_{k}\right)^{2}}{2 \sigma_{k}^{2}}}
$$

Clearly, we now have a statement that tells us how correcting $\vec{X}$ in a certain way depends on both the covariance matrix $\hat{C}$ and the DOCA vector $\delta \vec{X}$, thus meeting our immediate goal. The most illustrative way to proceed is to geometrically characterize our probability distribution. If we take an isoprobable surface, we will obtain a five-dimensional ellipsoid whose principal axes are oriented along the vectors $\vec{v}_{k}$, which are themselves, recall, mixtures of various DOCA variables. But what if we want to know the distribution a certain matrix will give for a pure DOCA variable, or some other general non-eigenmixture of variables? This is pretty simple if we force $\delta \vec{X}$ to be of the form $\xi \vec{u}$, where $\vec{u}$ is a vector in the direction of the combination whose distribution we're interested in. Permitting this substitution into Equation 13 and factoring out $-\xi^{2}$, we obtain by inspection the following effective distribution for a combination represented by $\vec{u}$ :

$$
\sigma_{\vec{u}}=\left(\sum_{k=1}^{5} \frac{\left(\vec{v}_{k} \cdot \vec{u}\right)^{2}}{\sigma_{k}^{2}}\right)^{-\frac{1}{2}}
$$

Now that we can say with certainty how a certain matrix is effectively smearing some variable, say $\Omega$, we can begin to figure out how to change this distribution without affecting the desirable mixing properties of the covariance matrix. First of all, an object describing the error mixing properties of the covariance matrix must be identified so that we may ensure its invariance. This is done by decomposing a covariance matrix $\hat{C}=C_{i j}$ in the following way: 


$$
C_{i j}=\sqrt{C_{i i}} \sqrt{C_{j j}} \Upsilon_{i j}
$$

The matrix $\Upsilon_{i j}=\hat{\Upsilon}$ is the one describing the mixing, and as such we may not touch it; hence we only are allowed to change the elements under the radicals. Since we are mainly going to be worried about forcing distributions on pure DOCA variables, we are further constrained to modify only the specific element under the radical corresponding to the variable of interest. For instance, if we're looking to impose a resolution on $\tan \lambda$, we only are allowed to modify $C_{44}$. So how does changing a single element in the decomposition in Equation 15 affect the effective distribution calculated in Equation 14? Attacking this final, central question analytically is unnatural and cumbersome, and the simple answer can be much more easily obtained by thinking about the problem geometrically. In order to do this, we can rewrite the decomposition in Eq. 15 in matrix form, allowing the matrix $\hat{\Delta}$ to have diagonal elements $\sqrt{C_{i i}}$ and off-diagonal elements zero:

$$
\hat{C} \rightarrow \hat{\Delta} \hat{\Upsilon} \hat{\Delta}
$$

We now allow ourselves to modify the $j$ th element of $\hat{\Delta}$ by letting a certain $\Delta_{j j} \rightarrow \sqrt{a} \Delta_{j j}$. This can be represented again as a set of matrix operations by introducing a new diagonal matrix $\hat{A}$ whose single nonunity diagonal element is $a$, at the intersection of the $j$ th row and column. This leads to the identification of the modified covariance matrix $\hat{C}^{\prime}$ with the decomposition:

$$
\hat{C}^{\prime} \rightarrow \hat{A} \hat{\Delta} \hat{\Upsilon} \hat{\Delta} \hat{A}
$$

Beginning our geometric analysis, we realize first that there is a 
certain probability $\widetilde{P}$ whose corresponding isoproabable surface is an ellipsoid with axes whose lengths are themselves the standard deviations for the distribution, $\sigma_{i}$; in analogy with the onedimensional distribution this probability $\widetilde{P}$ is shown to be $1 / \sqrt{e}$ times the normalization constant for the function. Hence, calculating distributions of variables is effectively nothing more than measuring "radii" of a certain five-dimensional ellipsoid in various directions. The next necessary realization is that physical equations describing isoprobable surfaces can be obtained by contracting the covariance matrix itself with $\delta \vec{X}$ enough times and setting that contraction equal to some appropriate constant:

$$
(\delta \vec{X})^{T} \hat{C} \delta \vec{X}=c
$$

By plugging in our decompositions of $\hat{C}$ and $\hat{C}^{\prime}$ into the above equation we obtain:

$$
\begin{gathered}
(\delta \vec{X})^{T} \hat{\Delta} \hat{\Upsilon} \hat{\Delta} \delta \vec{X}=c \\
(\delta \vec{X})^{T} \hat{A} \hat{\Delta} \hat{\Upsilon} \hat{\Delta} \hat{A} \delta \vec{X}=c
\end{gathered}
$$

We can allow the matrices $\hat{A}$ in the second equation above to act on the vectors $\delta \vec{X}$ rather than on the matrices, calling the resulting vectors $\delta \vec{X}^{\prime}$. These can be interpreted as vectors belonging to a new coordinate system whose $j$ th component has been dilated. It takes little convincing to see that the width of an ellipse along a coordinate $\delta X_{i}$ which has been stretched by a factor $\sqrt{a}$ will likewise be stretched by a factor $\sqrt{a}$; furthermore, since the deviations of the full distribution correspond exactly to the widths of a particular isoprobable surface, we can say that letting $\hat{C} \rightarrow \hat{C}^{\prime}$ 
as above will cause $\sigma_{\vec{u}} \rightarrow \sigma_{\vec{u}}^{\prime}=\sqrt{a} \sigma_{\vec{u}}$. The problem is therefore solved, with the needed multiplier a given by:

$$
a=\frac{\sigma_{\text {desired }}^{2}}{\sigma_{\text {original }}^{2}}
$$

where the deviations are both for the variable of interest. Using this method, we accomplish what we set out to do: we can give the user of the program the same kind of direct access to tracker resolutions as he has to calorimeter resolutions all while preserving the covariance of the tracker smearing method (even though it takes a lot of work to do and a lot of paper to explain).

\section{Performance}

Before releasing any program as a tool intended for general use, thorough testing and characterization must be carried out. In order to test the fast Monte Carlo simulator, a somewhat physically artificial stdhep file was written in order to perform debugging and characterization studies; in it an electron-positron pair annihilates to produce an up quark and an anti-up quark, each at exactly $250 \mathrm{GeV}$. Included in the characterization below performed using this file is an exhaustive group of figures with commentary included demonstrating the capabilities of the simulator at firstly simulating high energy physics events and secondly at extracting meaningful information on how physical results depend on basic detector performance characteristics. In brief, we first show raw particle-by-particle smearing histograms for the set of standard detector parameters, then we show that changing these parameter changes the smearing statistics as advertised in the implementation section. Second we show the results of a simple thrust analysis on the simulated $250 \mathrm{GeV} u \bar{u}$ events, followed by a study on how changing the detector parameters changes these physical results. Finally, in the last section of the 
paper, we perform a simulated measurement of the mass of the $Z_{0}$ boson, lightly touching on the dependence of the resolution of this measurement on the detector parameters.

\subsection{Raw Smearing Results}

The standard sdjan03 detector for the SLAC ILC group currently uses the following fast Monte Carlo parameters:

\begin{tabular}{|l|r|}
\hline Electromagnetic Calorimeter Onset: & $.1 \mathrm{GeV}$ \\
EM Cal. Sharpness: & $10.0 \% / \mathrm{GeV}$ \\
EM Cal. Minimum: $\theta$ : & $\cos ^{-1} .95$ \\
EM Cal. Energy Resolution Parameter $a$ : & .18 \\
EM Cal. Energy Resolution Parameter $b:$ & 0.0 \\
EM Cal. Position Resolution Parameter $a:$ & .10 \\
EM Cal. Position Resolution Parameter $b:$ & 0.0 \\
\hline \hline Hadronic Calorimeter Onset: & $.2 \mathrm{GeV}$ \\
H Cal. Sharpness: & .50 \\
H Cal. Minimum $\theta$ : & .08 \\
H Cal. Energy Resolution Parameter $a:$ & .10 \\
H Cal. Energy Resolution Parameter $b:$ & 0.0 \\
H Cal. Position Resolution Parameter $a:$ & $\cos ^{-1} .95$ \\
H Cal. Position Resolution Parameter $b:$ & $.2 \mathrm{GeV} / \mathrm{c}$ \\
\hline \hline Tracker Minimum Transverse Momentum: & \\
\hline \multicolumn{2}{|c|}{ Normal Tracker Covariance Matrices Used } \\
\hline
\end{tabular}

Shown below, in Figures 2 and 3, is a set of histograms showing the raw particle-by-particle smearing of the final-state particles in the stdhep file (henceforth called history particles) to the reconstructed particle content in the output slcio file using the unmodified sdjan03 detector.

\footnotetext{
${ }^{1}$ For definitions and implementation of the above variables, see the previous section.
} 

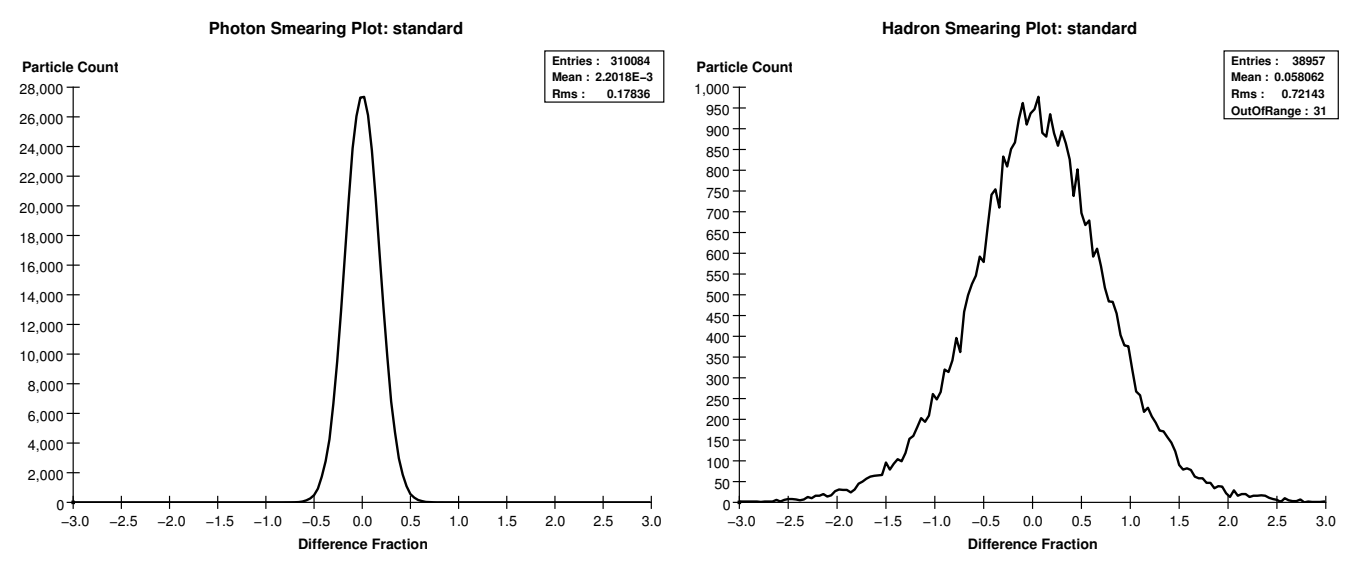

Figure 2: The above plots are histograms of the quantity $\left(E_{\text {recon }}-E_{\text {hist }}\right) / \sqrt{E_{\text {recon }}}$ binned for each photon (left) and hadron (right) occurring in a sample of $10,000 e^{+} e^{-} \rightarrow u \bar{u}$ events.
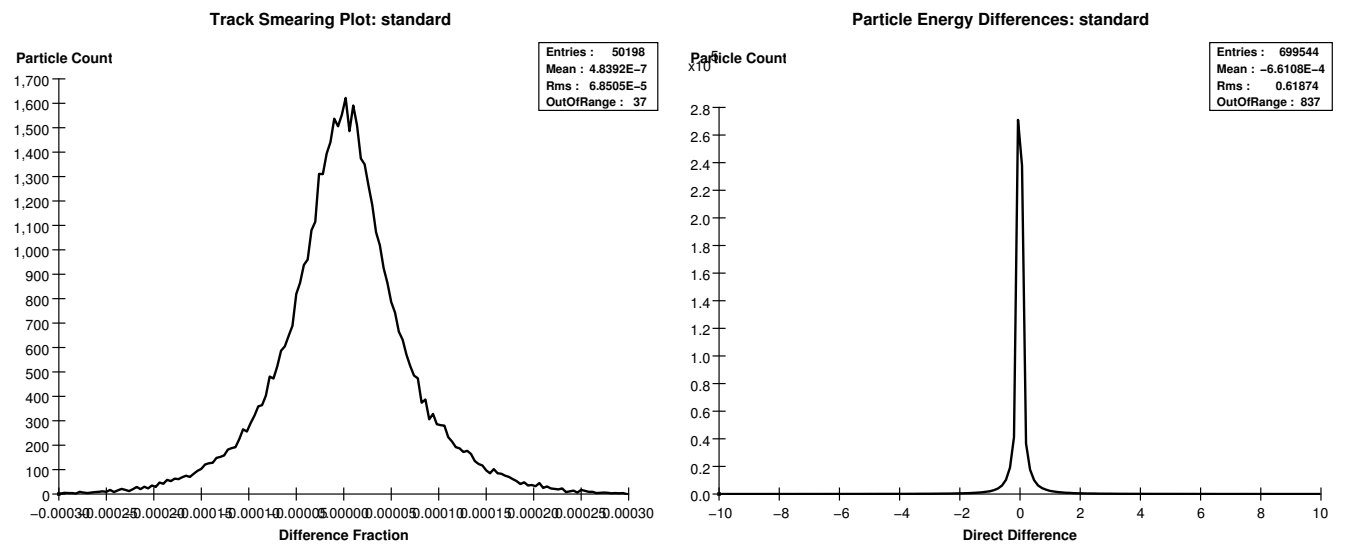

Figure 3: The above left histogram is the quantity $\left(P t_{\text {recon }}-P t_{\text {hist }}\right) / P t_{\text {hist }}^{2}$ where $P t$ is the transverse momentum binned for each charged particle, and the above right histogram is $E_{\text {recon }}-E_{\text {hist }}$ for each particle of any type that the detector finds.

Upon examining these histograms, the program certainly appears to be smearing as described in the implementation section, though there are a few discrepant features of interest in the figures. Clearly the photon distribution and the hadron distributions above have non-negligible mean values; in the hadronic plot this is due mostly to the fact that the offsetting $b$ parameter for 
the hadronic calorimeter is nonzero, but both have another significant error contribution. The source of this contribution lies in the fact that the program smears the energies of the particles in a gaussian fashion, for which there is a non-zero probability of smearing the particle's energy below its own mass. When this happens, the program simply draws another gaussian random number, effectively cutting off the distribution's tail below the particle mass, consequently modifying the error function for the distribution. Hence, for a particle of mass $m$, the error function is modified in the following way:

$$
\operatorname{erf}^{*}(E)=\left\{\begin{array}{cc}
\frac{\operatorname{erf}(E / \sqrt{2} \sigma)-\operatorname{erf}(m / \sqrt{2} \sigma)}{1-\operatorname{erf}(m / \sqrt{2} \sigma)} & E>m \\
0 & E \leq m
\end{array}\right.
$$

where the error function we're using is for a normalized distribution centered on $E_{\text {hist }}$, having domain $(0,1)$ and $\operatorname{erf}\left(E_{\text {hist }}\right)=\frac{1}{2}$. These details are complicated by the fact that in our particular choice of event, many more sensitive, low energy photons are created than high energy ones, compounding the effect. Having spoken to this issue, we shall explore the effects of changing the electromagnetic calorimeter parameter $a$ on the shapes of the smearing histograms, just to ensure that the program is actually smearing energies to model our detector input. Setting $a=.09$ and letting the rest of the parameters remain as standard produces the histograms found in Figure 4 .

Notice in Figure 4 that the hadronic distribution is identical to that found in Figure 2, as expected. Also note that the non-zero mean discussed above is decreased by about an order of magnitude, which is in line with the interpretation of this mean's non-zero divergence given in the comments following Figure 2. Next, in Figures 5 and 6, are the histograms that follow from setting first $a=.27$ then lastly $a=.36$.

Again, the most notable features in Figures 5 and 6 are the small nonzero means in the photon smearing plots; notice that the deviation from zero varies approximately exponentially as $a$ is changed, achieving ten percent of 

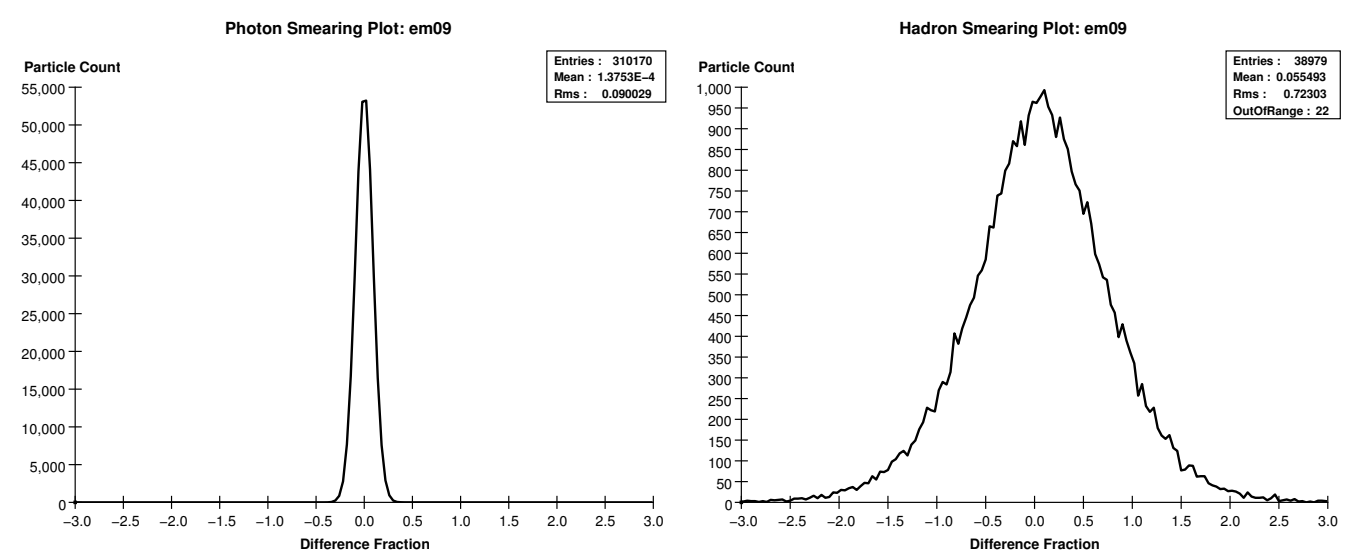

Figure 4: The above plots are histograms of $\Delta E / \sqrt{E}$ for each photon (left) and hadron (right) in the standard detector, but with electromagnetic calorimeter parameter $a$ set to .09 .
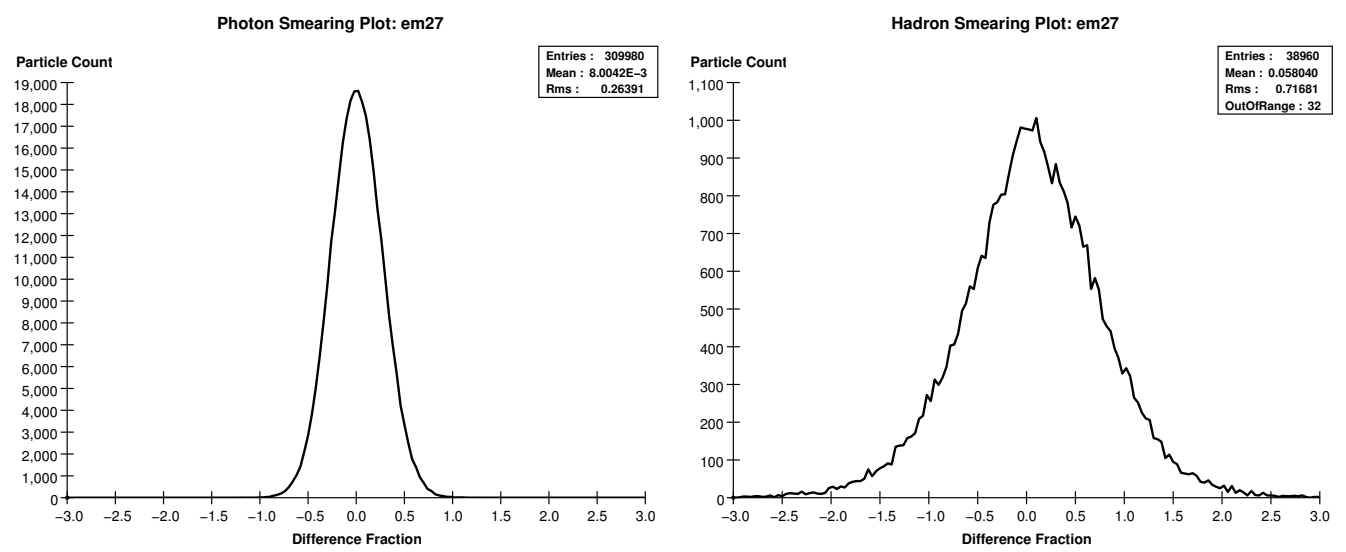

Figure 5: The above plots are histograms of $\Delta E / \sqrt{E}$ for each photon (left) and hadron (right) in the standard detector, but with electromagnetic calorimeter parameter $a$ set to .27 .

the deviation in the final histogram where $a=.36$. Reassuringly we have a very clean, straightforward dependence for the electromagnetic calorimeter deviation on the supplied parameter $a$; in short, we can safely conclude that this aspect of the program is working properly.

Now, let us turn our attention to the hadronic resolution smearing parameter, which, recall, is implemented in an identical fashion to the correspond- 

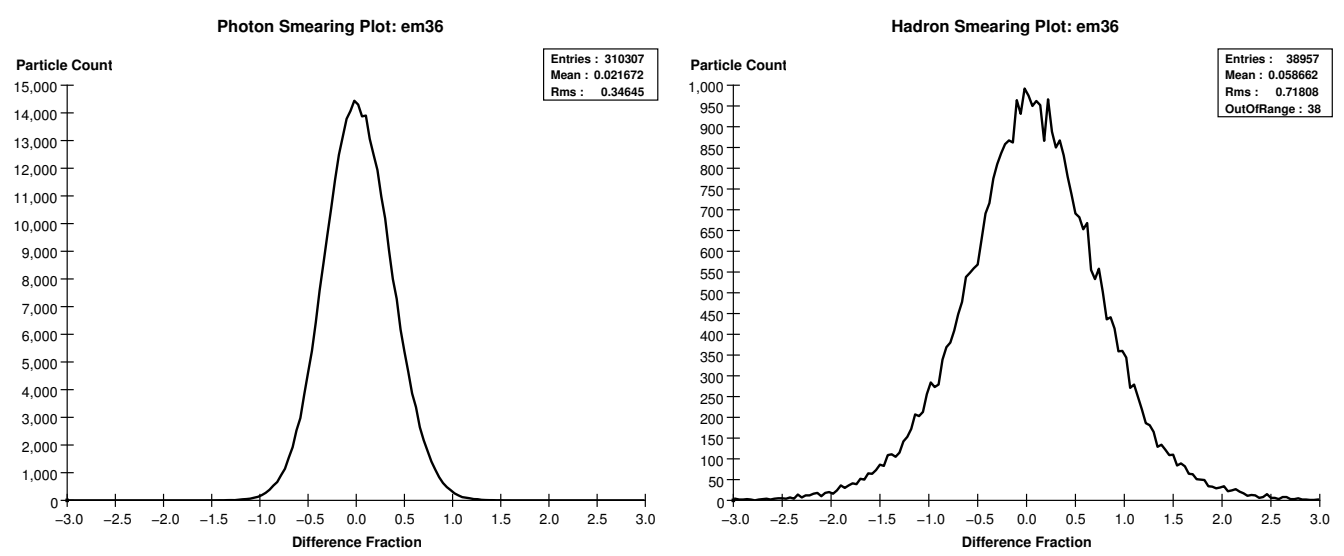

Figure 6: The above plots are histograms of $\Delta E / \sqrt{E}$ for each photon (left) and hadron (right) in the standard detector, but with electromagnetic calorimeter parameter $a$ set to .36 .

ing electromagnetic calorimeter parameter. The main difference between how these two types particles are smeared lies in the error-function correction effect mentioned above ${ }^{2}$, to which the hadrons are more sensitive due to their significant masses. Nevertheless, the effect of changing the resolution parameter is very similar to that seen in the photonic distributions by changing the corresponding electromagnetic calorimeter parameter; this can be seen in the following histograms, for the first of which $a=.30$ in Figure 7 .

The salient features here are again the mean and the standard deviation, now of the hadronic distribution on the right. Contrast the mean of the standard detector hadronic distribution $(\sim .05$, found in Figure 2$)$ with Figure 7 ?s mean value of about .02. This decrease in mean divergence is isomorphic to that which occurs in the photonic distribution when the electromagnetic calorimeter $a$ is dropped from .18 to .09. The hadronic mean, in keeping with the behavior of the photonic plot, diverges significantly as the resolution is made worse; this effect can be seen in Figure 8, for which $a=.70$.

Clearly, as seen in Figure 8, the mean offset issue is much more significant for massive particles (as compared to photons) when the energy resolution is

\footnotetext{
${ }^{2}$ see page $\mathrm{XX}$.
} 

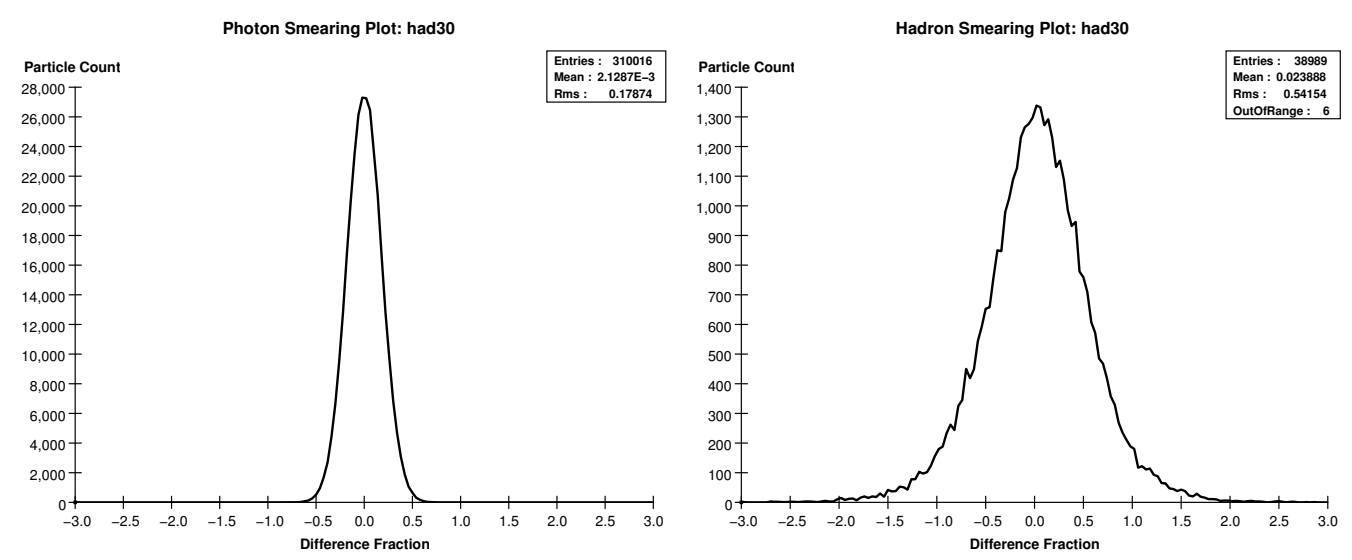

Figure 7: The above plots are histograms of $\Delta E / \sqrt{E}$ for each photon (left) and hadron (right) in the standard detector, but with hadronic calorimeter parameter $a$ set to .30 .
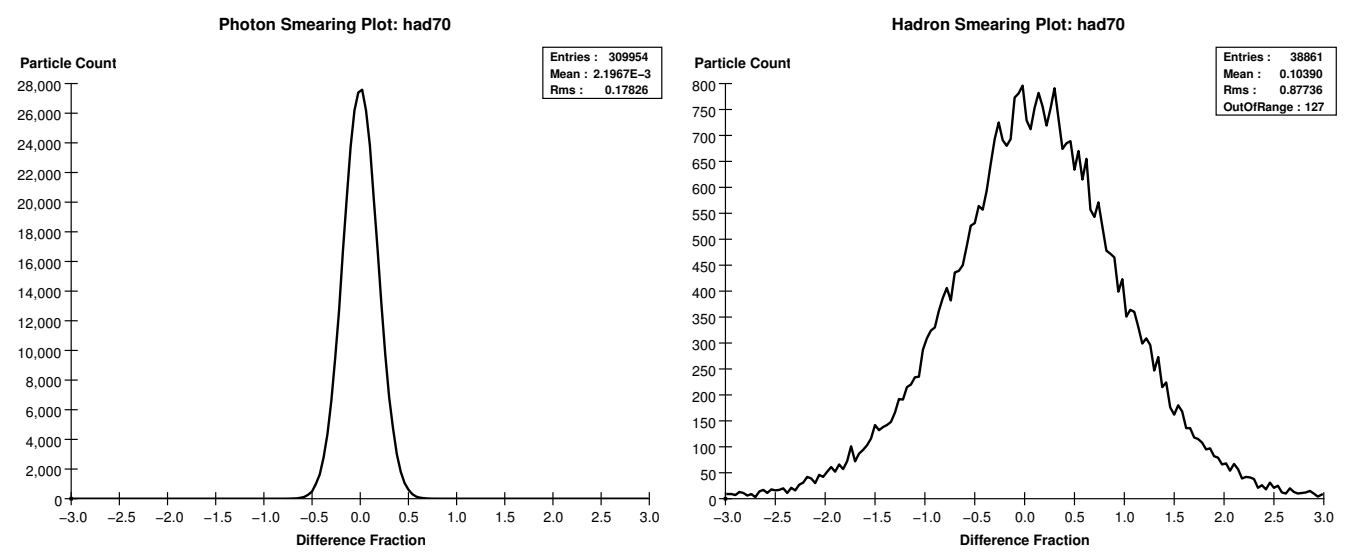

Figure 8: The above plots are histograms of $\Delta E / \sqrt{E}$ for photons (left) and hadrons (right) in the standard detector with the hadronic calorimeter parameter $a$ set to .70 .

made very bad, indicating the sensitivity of the function $\operatorname{erf}^{*}(E)$ to changes in the resolution when $\operatorname{erf}(m)$ is significantly large.

Finally, let us turn to the performance of the modified tracking parameterization; it is especially important to check the implementation of this feature as its derivation was quite complicated and because it is the only practical way we can directly modify the way the tracker is modeled. A very 
useful choice of tracker parameterization (we will want to use our modified tracker smearing method to force the curvature parameter in order to change the energy resolution, of course) is the following:

$$
\Delta \Omega \propto \sqrt{(a)^{2}+\left(\frac{b}{P t \cdot \cos \theta}\right)^{2}}
$$

where the constant of proportionality is composed of factors of $\Omega$ and $p_{\text {track }}$ inserted to correct the units. A nice feature about programming in the modified tracker control is that it gives us great flexibility in not only our choice of specific parameters, but also in our choice of parameterization function itself. Using the above equation for $\Delta \Omega$, a choice for the tracker parameters giving very reasonable distributions is $a=2.1 \times 10^{-5}$ and $b=1.0 \times 10^{-4}$; this is the ?simple-standard? distribution, and the histograms generated using these choices of parameter appear below, in Figure 9.
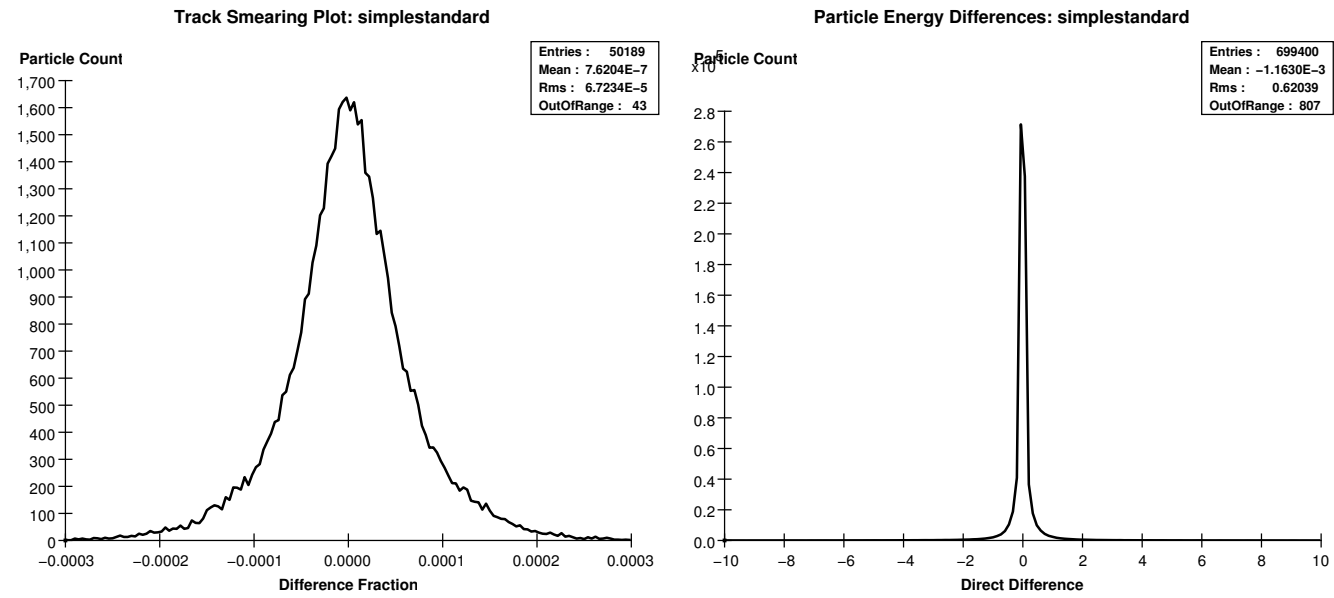

Figure 9: The plots above were generated by forcing $\Delta \Omega$ as described above, in Eq. 22, with $a=$ $2.1 \times 10^{-5}$ and $b=1.0 \times 10^{-4}$. The histograms are of the quantity $\left(P t_{\text {recon }}-P t_{\text {hist }}\right) / P t_{\text {hist }}^{2}$, again track-by-track over the first 10,000 events in the $u \bar{u}$ stdhep file where $P t$ is the transverse momentum of the track. 
It is not immediately apparent in Figure 9, but the tracker smearing plot on the left is (as the parameterization of the curvature resolution introduced above might suggest) a collapsed image of a bivariate distribution that depends both on energy and on the angle $\theta$ of the tracks; as such it will exhibit some very interesting properties as we vary $a$ and $b$ in Equation 22 . The distribution itself is only approximately gaussian at the core; the large tails on the distribution betray that it would be more accurately fitted to $e^{-\sqrt{a^{2}(E-b)^{2}+c^{2}}}$ (an exponentiated hyperbola). A quick comparison with the standard detector tracker distribution in Figure 3 justifies our decision to call these values of $a$ and $b$ the standard set, as the difference of the deviations of the two distributions is only $1.3 \times 10^{-6}$. Let us now vary the $a$ parameter and observe the changes incurred in the distribution for tracking momentum. Below are histograms constructed by letting $a=1.0 \times 10^{-5}, 4.0 \times 10^{-5}$, and $8.0 \times 10^{-5}$, contained in Figures 10-12.
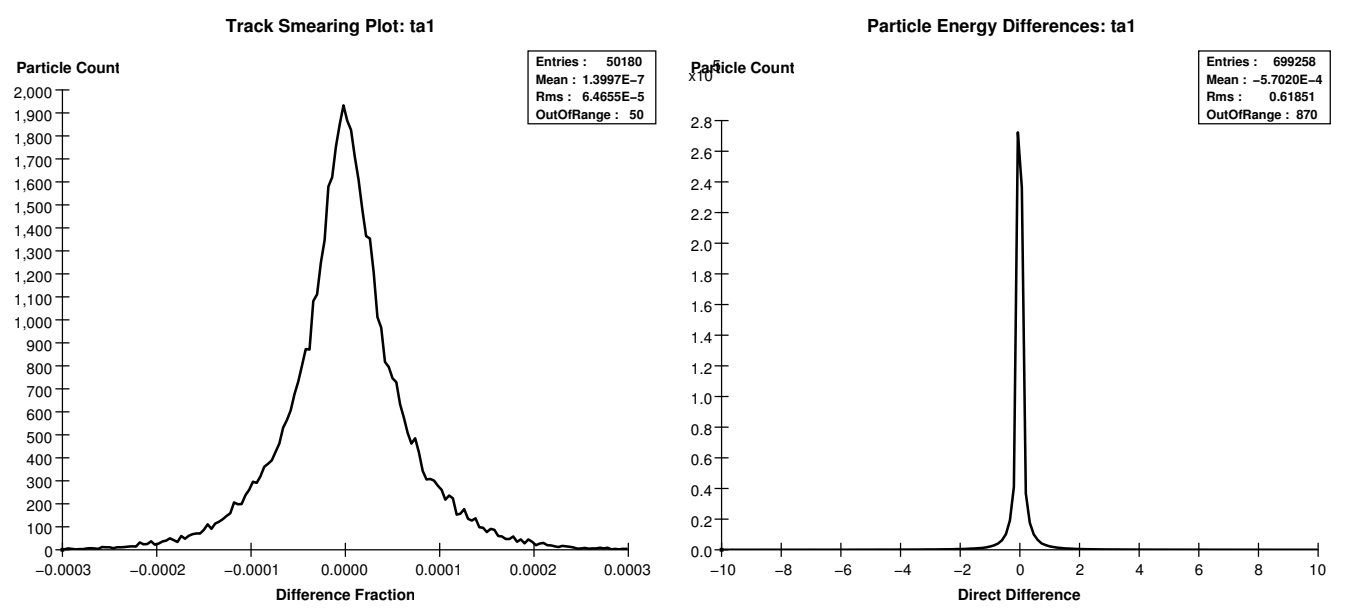

Figure 10: The above left histogram is the quantity $\Delta P t / P t^{2}$ (where $P t$ is the transverse momentum) generated with tracker parameter $a$ (see Eq. 22 ) set to $1.0 \times 10^{-5}$ and $b$ left at its simple-standard value.

In Figures 10-12, the previous comment on the tracker histogram being a collapsed projection of a bivariate distribution gains a great deal of meaning, with manifestly concrete consequences. First of all, we see in the histogram 

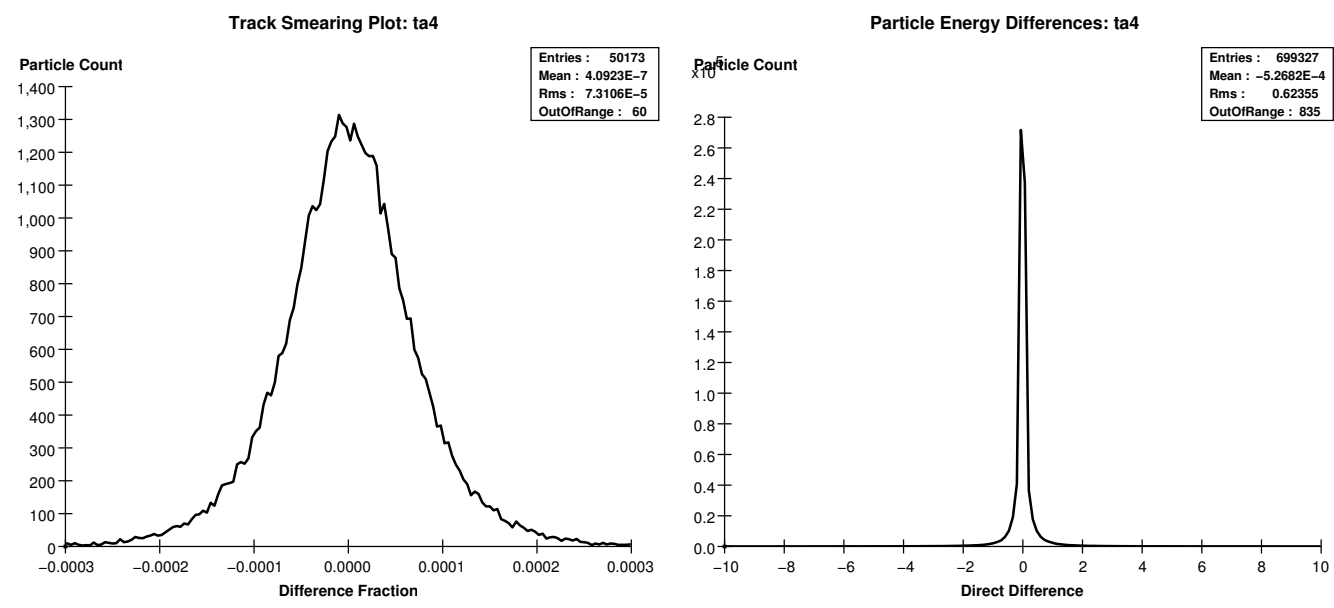

Figure 11: The above left histogram is the quantity $\Delta P t / P t^{2}$ (where $P t$ is the transverse momentum) generated with tracker parameter $a$ (see Eq. 22 ) set to $4.0 \times 10^{-5}$ and $b$ left at its simple-standard value.
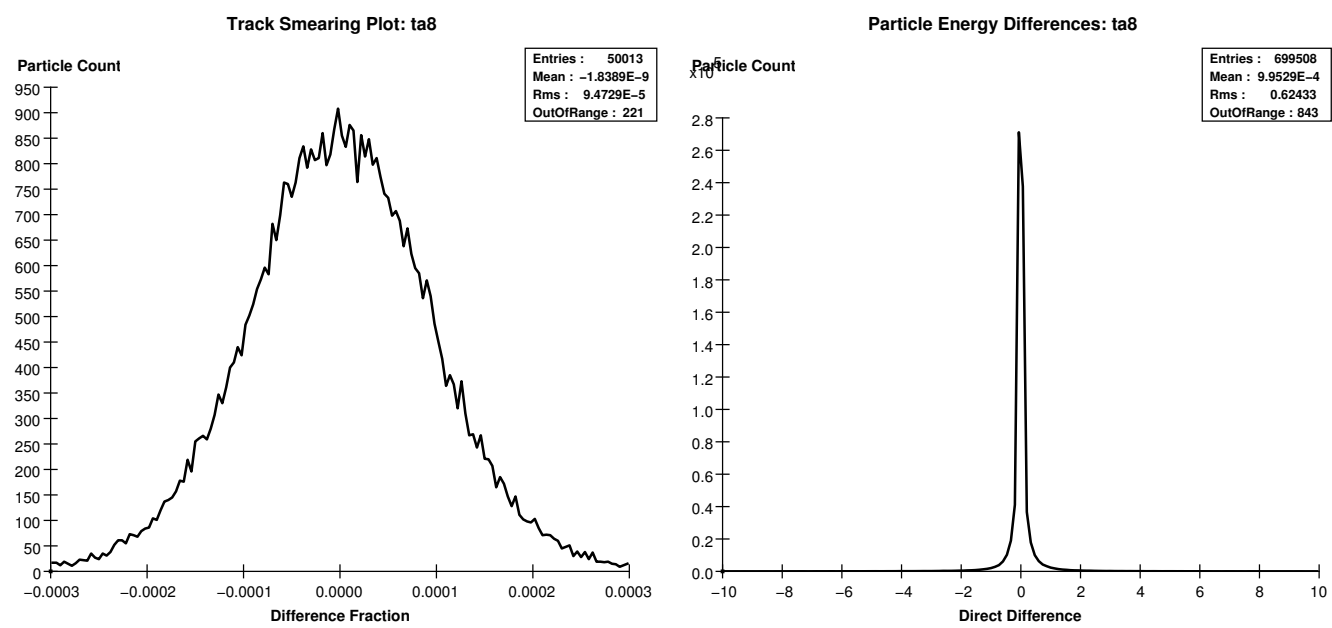

Figure 12: The above left histogram is the quantity $\Delta P t / P t^{2}$ (where $P t$ is the transverse momentum) generated with tracker parameter $a$ (see Eq. 22) set to $8.0 \times 10^{-5}$ and $b$ left at its simple-standard value.

for which $a=1.0 \times 10^{-5}$ (Figure 10) that the core of the histogram is much more sharply peaked than in Figure 9; If the distribution were in fact gaussian we'd expect a situation where the derivative of the distribution smoothly 
crosses zero at the mean, but instead we get a situation where the resulting histogram is quite "pointy", elucidating the fact that we're only allowed to glimpse a shadow of what is really happening. Looking at the other end of the resolution spectrum in Figures 11 and 12, we see that the opposite happens. The core of the distribution gets much blurrier, with the top smoothing out and gaining statistical fuzziness; generally the hits are less localized around a clear mean as $a$ increases. These are all very good signs that the smearing procedure is working as designed, and certainly we can, if we wish, arbitrarily let $a$ change until we get a distribution that looks appropriate for whatever purpose. Now let us turn our attention to the $b$ parameter, whose smearing term depends on $\cos \theta$. Again, we must bear in mind the multivariate nature of these distributions, and as we will see, the phenotype for changing the $b$ parameter is much different than that for changing $a$. To illustrate this, below are histograms constructed by letting $b=0.5 \times 10^{-3}, 2.0 \times 10^{-3}$, and finally $4.0 \times 10^{-3}$, contained in Figures $13-15$.
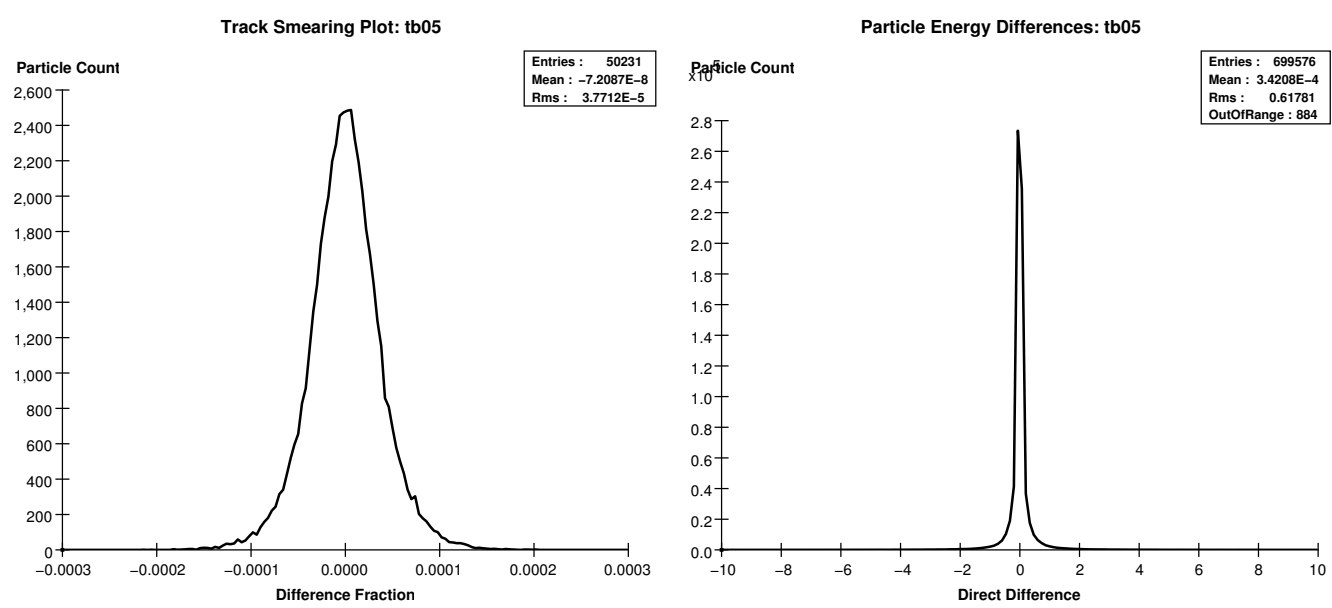

Figure 13: The above left histogram is the quantity $\Delta P t / P t^{2}$ (where $P t$ is the transverse momentum) generated with tracker parameter $b$ (see Eq. 22) set to $0.5 \times 10^{-3}$ and $a$ left at its simple-standard value.

As claimed, changing the parameter $b$ in Eq. 22, put bluntly, looks very different than does changing the parameter $a$. The parameter $b$ too physically 

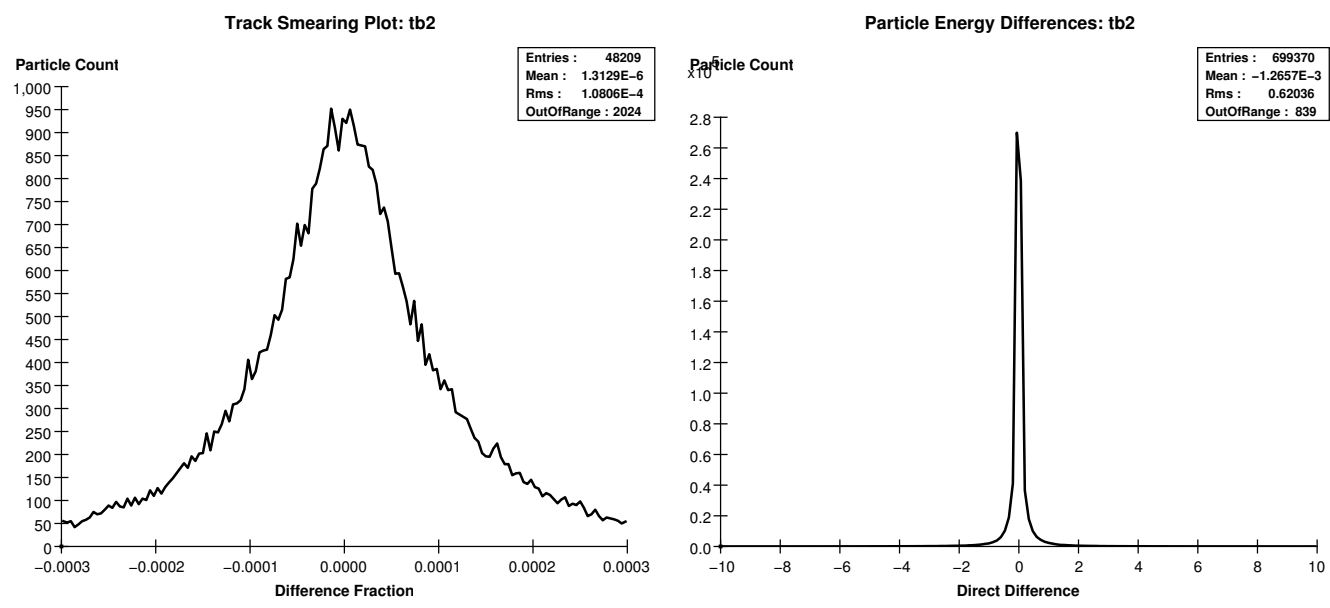

Figure 14: The above left histogram is the quantity $\Delta P t / P t^{2}$ (where $P t$ is the transverse momentum) generated with tracker parameter $b$ (see Eq. 22 ) set to $2.0 \times 10^{-3}$ and $a$ left at its simple-standard value.
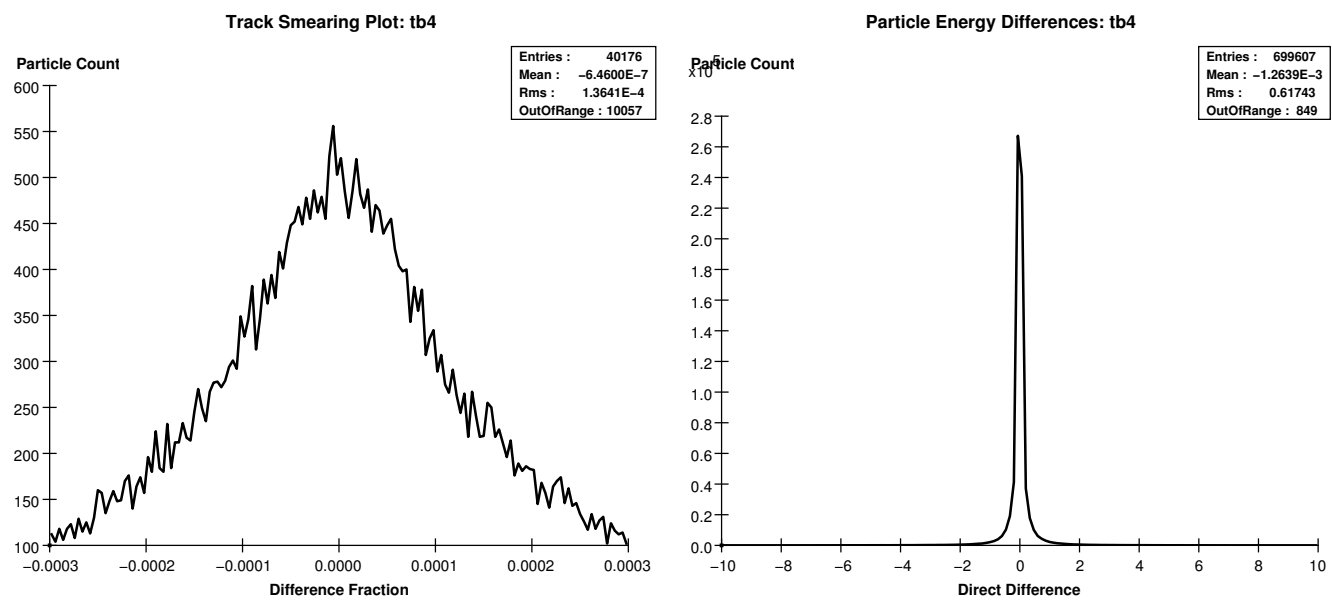

Figure 15: The above left histogram is the quantity $\Delta P t / P t^{2}$ (where $P t$ is the transverse momentum) generated with tracker parameter $b$ (see Eq. 22) set to $4.0 \times 10^{-3}$ and $a$ left at its simple-standard value. Note the vertical scale.

has a different role from that of $a$, describing how the accuracy in measuring the curvature is reduced as the track becomes more and more in line with the beam pipe. This is certainly a realistic dependence to expect, since as 
the angle $\theta$ decreases, so does the fraction of the total momentum that we're able to measure. This of course has the effect of amplifying the error in the measurement of the total momentum, which is the quantity of greatest interest here. We can see in the histograms for varied $b$ that increasing this parameter tremendously increases the significance of, primarily, the tail of the distribution, producing histograms that are decidedly non-gaussian. Nonetheless, we do achieve the expected overall effect, with the net resolution plummeting as $b$ is increased.

\section{2 uū Events at $500 \mathrm{GeV}$ : Thrust Analysis}

Our next task, having shown that the program is performing properly, is to assess how changing the detector parameters affects measurable physical quantities in a realistic event analysis. Since the stdhep file we?re using has each quark at $250 \mathrm{GeV}$, the event should be boosted enough to keep each quark jet relegated to one thrust hemisphere, just by conservation of

momentum. By calculating the thrust axis $\vec{T}$ for each event, we should be able to divide up all the particles in the detector as belonging to one quark or the other by determining the sign of $\vec{T} \cdot \vec{p}_{\text {recon }}$ for each reconstructed particle, where $\vec{p}_{\text {recon }}$ is the full 4-momentum. After performing the above analysis on both the set of reconstructed particles and the set of history particles, we can bin the differences between the energies summed up in each thrust hemisphere for each type of particle in order to get a histogram of the jet energy resolution for the detector. Since the measured jet energy contains measurements from the tracker and both calorimeters, the jet energy resolution is going to be a highly composite plot; thus it will have some very interesting behavior as the properties of the various components of the detector are changed. Determining this behavior is exactly the goal of the fast Monte Carlo; with our present experience we can already see how sensitively this is going to depend on the types of particles produced in the events we're studying, among other things. 
First of all, let us examine the results of such a thrust analysis performed on an slcio file generated using the standard detector description. Below, in Figures 16-18, are histograms of the measured jet energies for both the reconstructed particles and history particles, the total jet energy resolution, the photonic and hadronic jet energy resolutions, the charged jet energy resolution, and finally a plot showing measured photon energies.
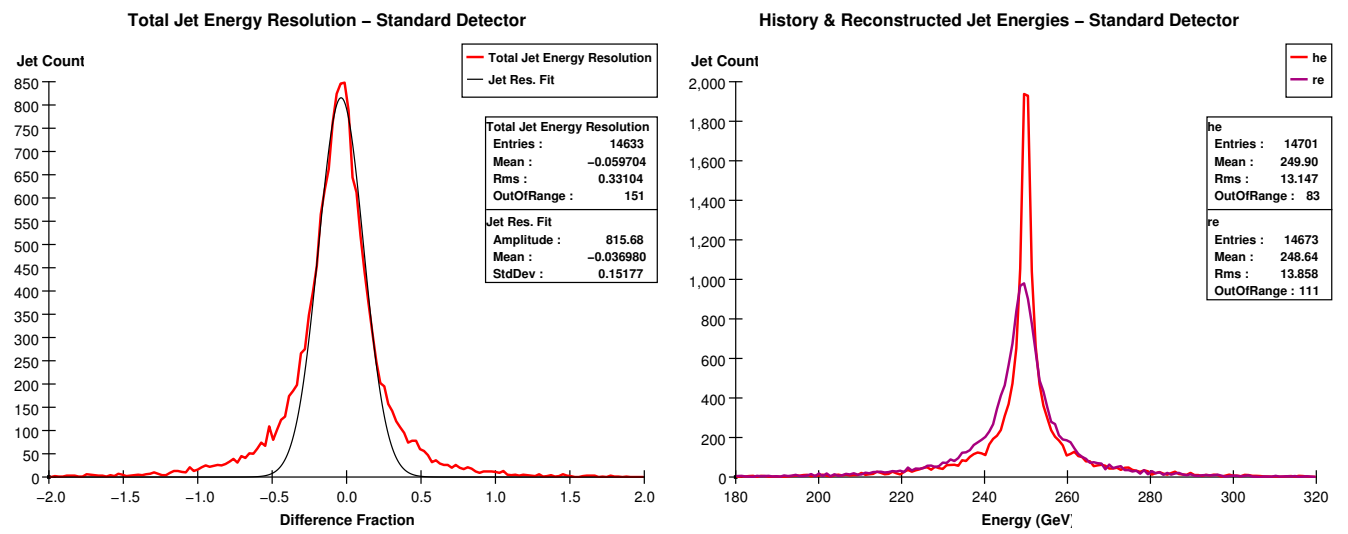

Figure 16: The above right histogram shows simply the measured jet energies for both the history particles and the reconstructed particles, whereas the left histogram shows the jet energy resolution, and is a binning of the quantity $\left(E_{\text {recon }}-E_{\text {hist }}\right) / \sqrt{E_{\text {hist }}}$ As the jet energy resolution for the detector, this is going to be the figure of primary interest.

In Figure 16 we see our first jet energy resolution plot, which is the quantity which will be of greatest interest to us in the remainder of the paper. Again, it's a highly composite distribution, with an only approximately gaussian shape. The fit that has been applied was calculated using a QR factorization, and for each jet energy resolution the fit will be calculated using only the core of the distribution, a decision justified firstly on the non-gaussian nature of the distribution and secondly on the fact that when looking for particle masses with this program, only the core of a mass peak is important. On the right we see the raw jet energy measurements; of greatest interest here are the losses that occur in the detector during the simulation process. 
This is the first indication we see of the efficiency function mentioned in the implementation section. We will look further into this loss by hermetically sealing the detector in the next group of histograms we analyze.
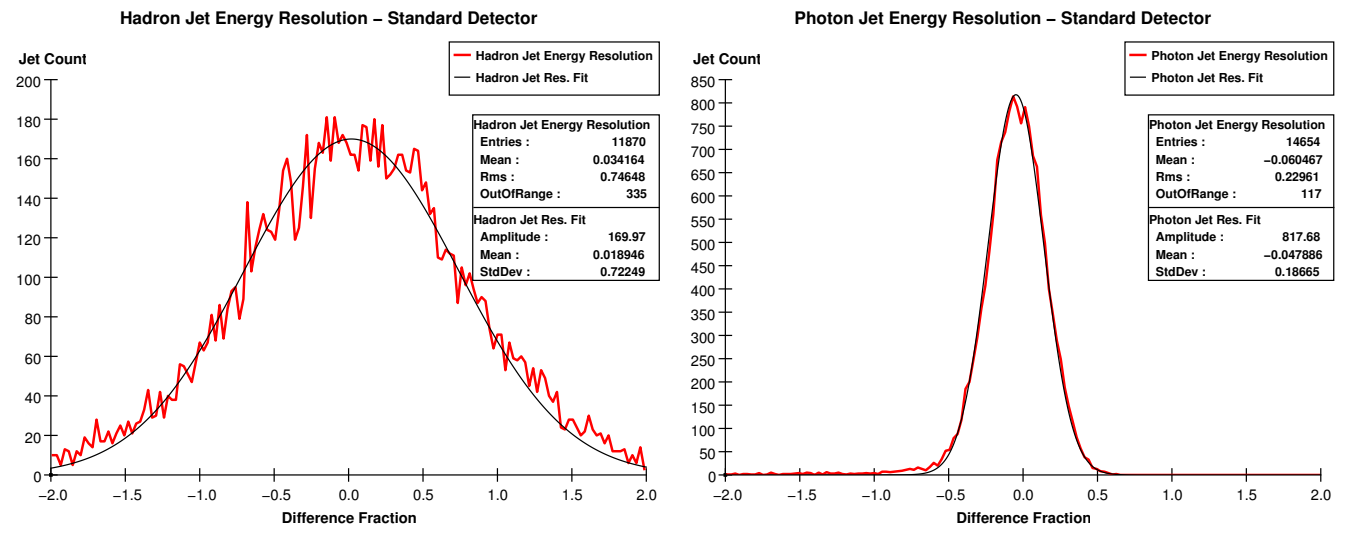

Figure 17: The above figure contains a plot of the photonic and the hadronic jet energy resolutions for the standard detector parameterization.

In Figure 17 we see the isolated photonic and hadronic components of the jet energy. These histograms will naturally be of direct interest when we start varying the parameters for the different calorimeters again in order to see what effect changing the resolution has on the total jet energy plot. Here too, albeit in a very muted way, we can see the non-hermeticity of the detector. This effect shows up as a tiny asymmetry in the tails of the photonic distribution; clearly a few more reconstructed jets are coming in at lower energy with respect to the history jets than at higher energy. Were our statistics higher, we could also observe this tail asymmetry effect in the hadronic energy resolution plot. Again, we can investigate this effect further by making the detector hermetic.

Finally, in Figure 18 we have a plot of the tracker energy resolution as well as a photon spectrum for the $u \bar{u}$ event. The tremendous negative tail on the tracker energy resolution makes fitting gaussians to this distribution especially problematic, but again we will see that this tail is completely due to 

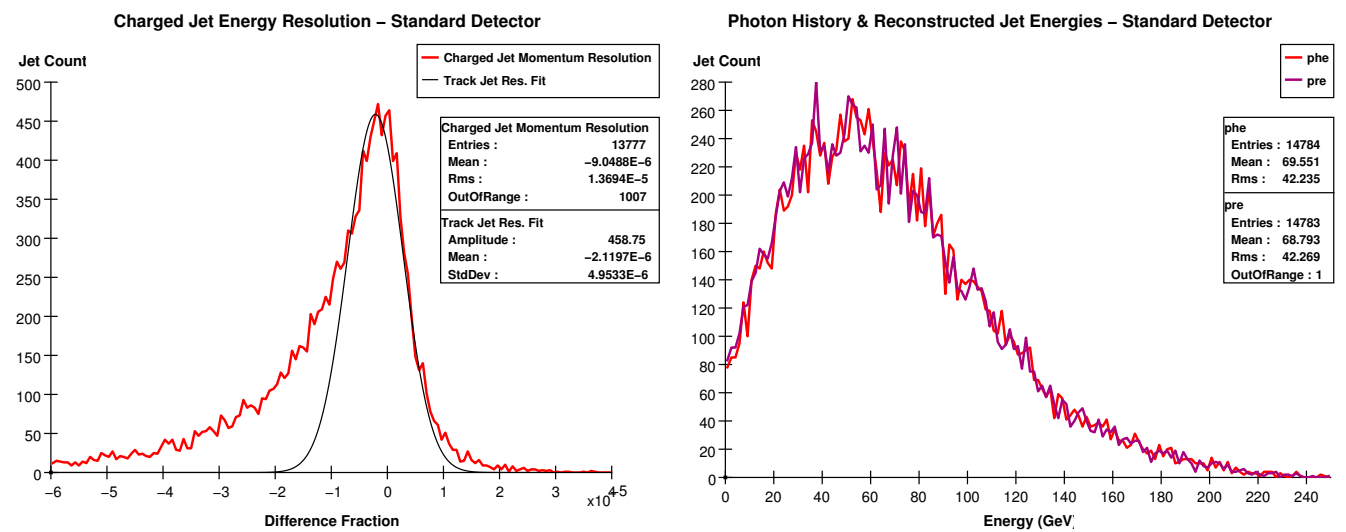

Figure 18: The above figure contains on the left a plot of the charged component of the jet energy resolution, whereas on the right appears a spectrum of the total jet energy contained in the photon radiation from the event.

particle escape or efficiency losses, and that this effect completely disappears when we make the detector hermetically sealed. On the right we see a plot of the photon spectrum, and notably, the total photon energy is more likely to be at the low-energy end of the spectrum. The total tracker spectrum (not shown) is much like the photon spectrum, only it is weighted more toward the high-energy end of the spectrum.

Now, after finally being able to observe effects due to particle loss, let us take a look at what occurs when we try to prevent this by making the detector perfectly efficient and removing all beam-pipe losses. We call such a detector configuration "hermetically sealed", which is almost true since we shall still let neutrinos escape. Below are the same histograms as appear in Figures 16-18, only now we will force different values for a few the parameters found in the table on page 22 : 


\begin{tabular}{|l|r|}
\hline Electromagnetic Calorimeter Onset: & $\begin{array}{r}.1 \mathrm{GeV} \rightarrow 0.0 \mathrm{GeV} \\
\text { EM Cal. Sharpness: }\end{array}$ \\
EM Cal. Minimum: $\theta:$ & $\begin{array}{r}10.0 \% / \mathrm{GeV} \rightarrow 10,000 \% / \mathrm{GeV} \\
\cos ^{-1} .95 \rightarrow \cos ^{-1} 1.00\end{array}$ \\
\hline Hadronic Calorimeter Onset: & $.2 \mathrm{GeV} \rightarrow 0.0 \mathrm{GeV}$ \\
H Cal. Sharpness: & $10.0 \% / \mathrm{GeV} \rightarrow 10,000 \% / \mathrm{GeV}$ \\
H Cal. Minimum $\theta:$ & $\cos ^{-1} .95 \rightarrow \cos ^{-1} 1.00$ \\
\hline Tracker Minimum Transverse Momentum: & $.2 \mathrm{GeV} / \mathrm{c} \rightarrow 0.0 \mathrm{GeV} / \mathrm{c}$ \\
\hline
\end{tabular}

The resulting histograms can be found below, in Figures 19-21.
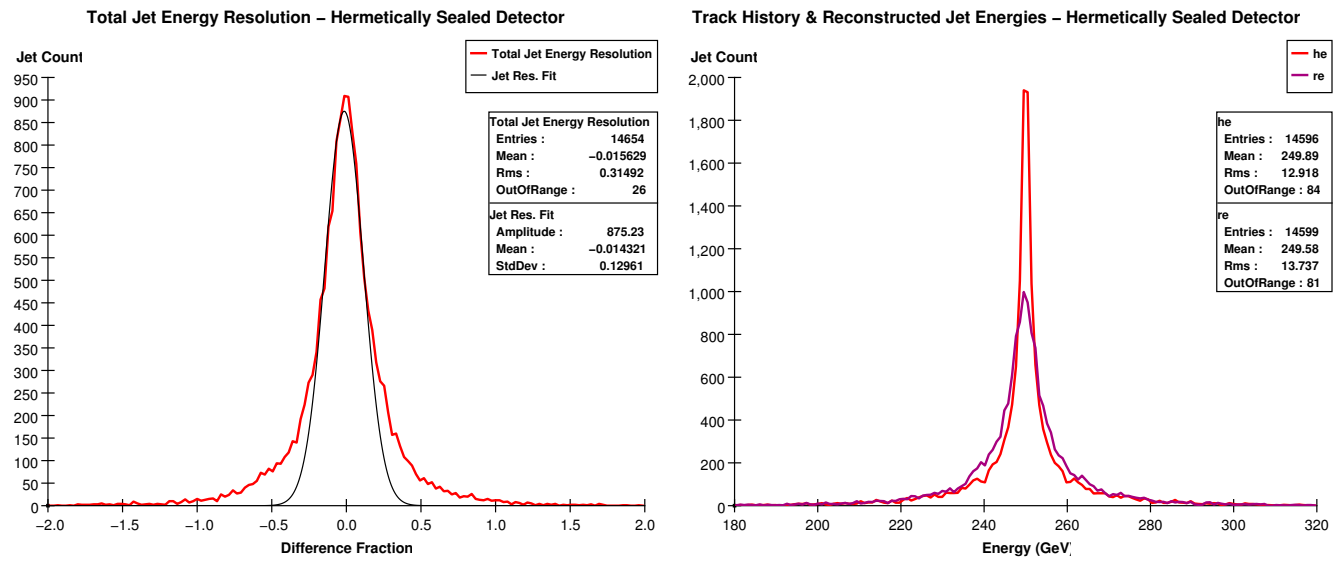

Figure 19: The above figure contains a plot of the total jet energy resolution and the raw history and reconstructed jet energies as generated by the hermetically sealed detector; this detector is described by the table on page 22 with the changes included in the table above.

The left plot in Figure 19 contains the total jet energy resolution after sealing the detector; upon comparison with the standard jet energy resolution in Figure 16 we see a marked improvement. (The fitted resolutions are $15.177 \%$ as compared to $12.963 \%$ ). Also, we see that the peak difference between the history particles and the reconstructed particles in the raw jet energy plot has appreciably disappeared.

Figure 20 contains the photonic and hadronic energy resolutions. Recall that the photonic energy resolution had a small negative tail in Figure 17; 

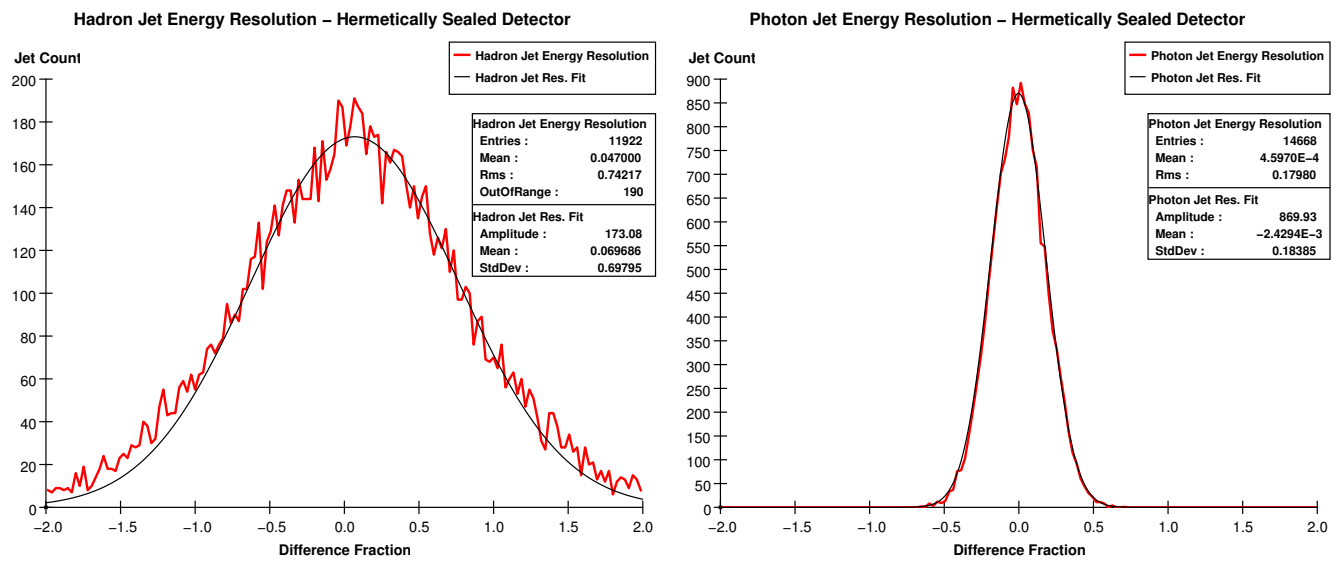

Figure 20: The above figure contains a plot of the photonic and the hadronic jet energy resolutions for the standard detector parameterization.

by sealing up the detector completely we were able to make this effect cease to appreciably exist. Also, notably, the resolution is not much improved at all: the standard detector is able to deliver a resolution of $18.665 \%$, while the sealed calorimeter delivers $18.385 \%$.
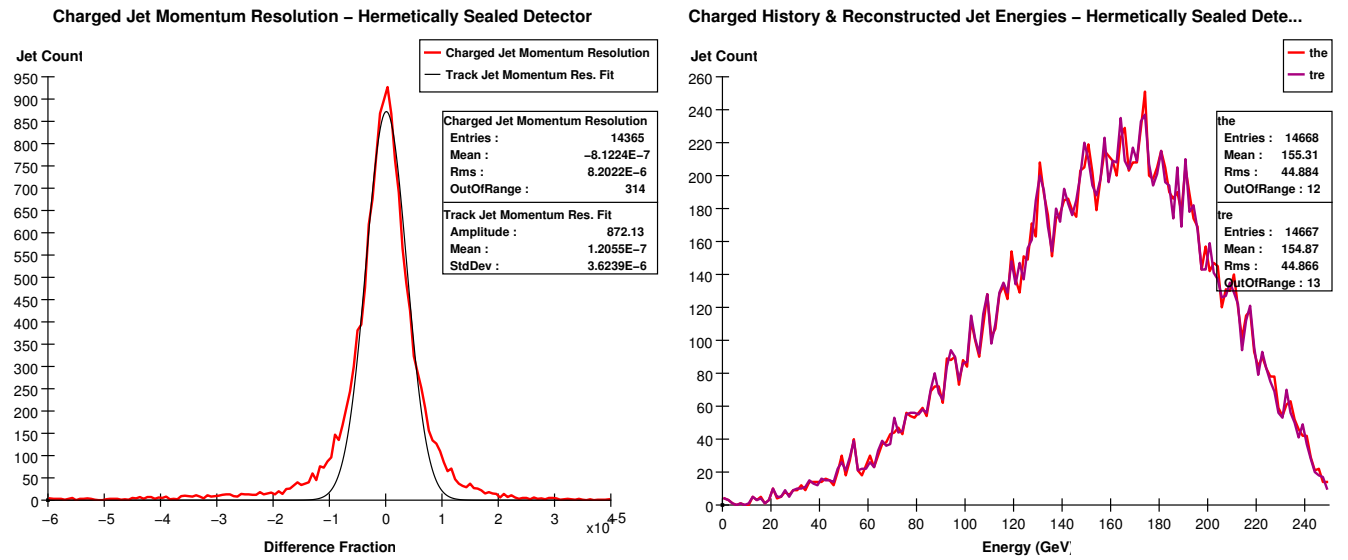

Figure 21: The above figure contains a plot of the photonic and the hadronic jet energy resolutions for the standard detector parameterization.

Finally, looking at Figure 21, we see a huge change in the appearance 
of the tracker energy resolution plot: the daunting negative tail found in the standard detector plot (Figure 18) has completely disappeared. From the changes we've observed in this set of data, we can confidently conclude that the offset from zero in the standard detector energy resolution is almost entirely due to loss/efficiency effects, and that among these the most significant contributor is the loss of particles in the tracker due to the transverse momentum minimum parameter.

Having established this important causal point on detector performance, let us now proceed with observing the dependence of the physics performance on the most important variables; the resolution parameters. This is the primary task this program will be used to complete, only our particular choice of event is probably the simplest type of event analysis possible to conduct while still achieving some level of realism. Below, in Figures 22-27, we can find a group of two pertinent thrust analysis figures for each of three different values of the electromagnetic resolution parameter $a$, followed by commentary. The values $a$ assumes are $a=.09, .27$, and .36 .
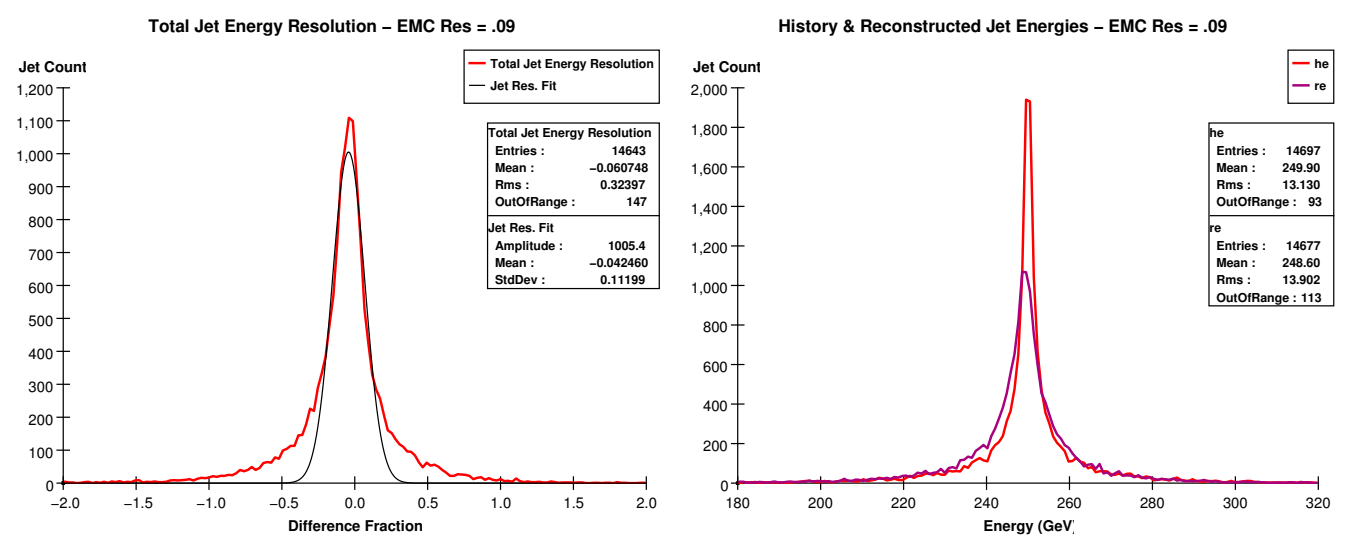

Figure 22: The above figure contains a plot of the photonic and the hadronic jet energy resolutions for the standard detector parameterization.

The histograms contained in Figures 22-27 themselves contain a great deal of important physics results. Since photons are a major component of 

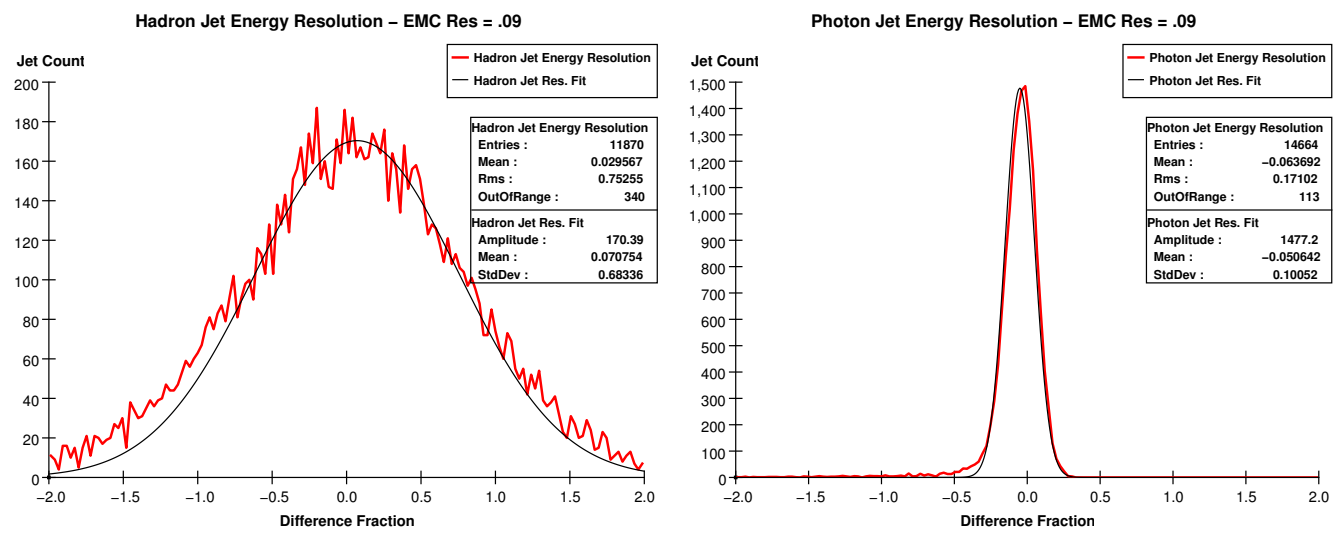

Figure 23: The above figure contains a plot of the photonic and the hadronic jet energy resolutions for the standard detector parameterization.
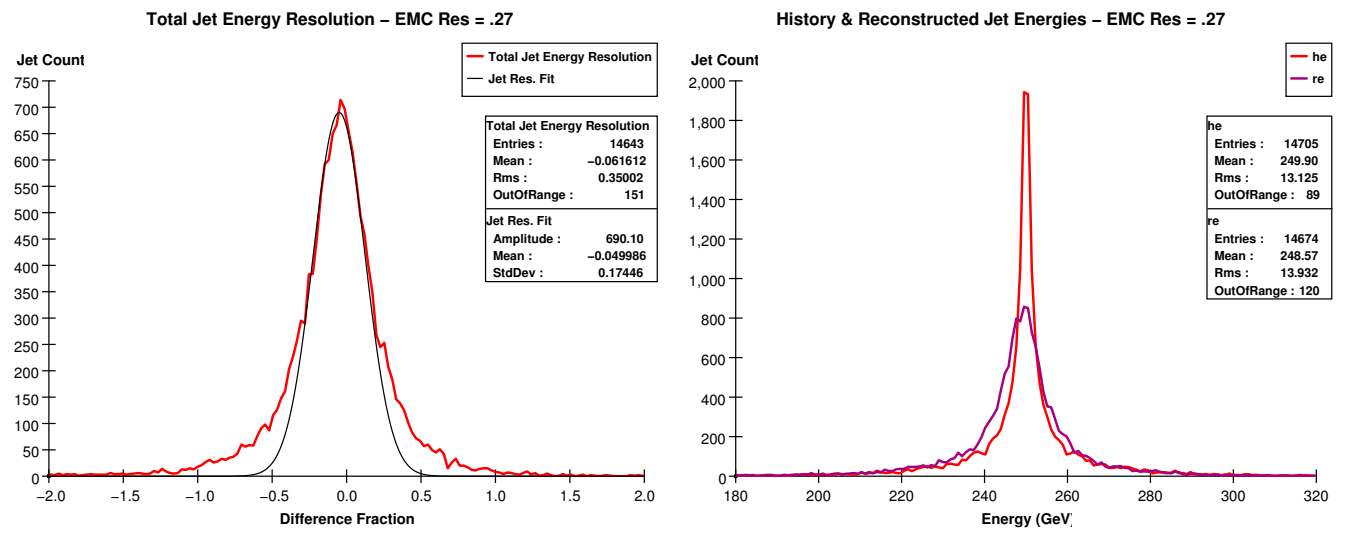

Figure 24: The above figure contains a plot of the photonic and the hadronic jet energy resolutions for the standard detector parameterization.

the $u$ quark jets we observe, we see that varying the electromagnetic calorimeter resolution has a significant effect on the total jet energy resolution. However, we can also see that the blurriness of the jet energy resolution does have a maximum limiting value (with respect to changes in the electromagnetic parameter $a$ ), as evidenced by the fact that the change in jet energy resolution between the standard value for $a$ and $a=.27$ is greater than the 

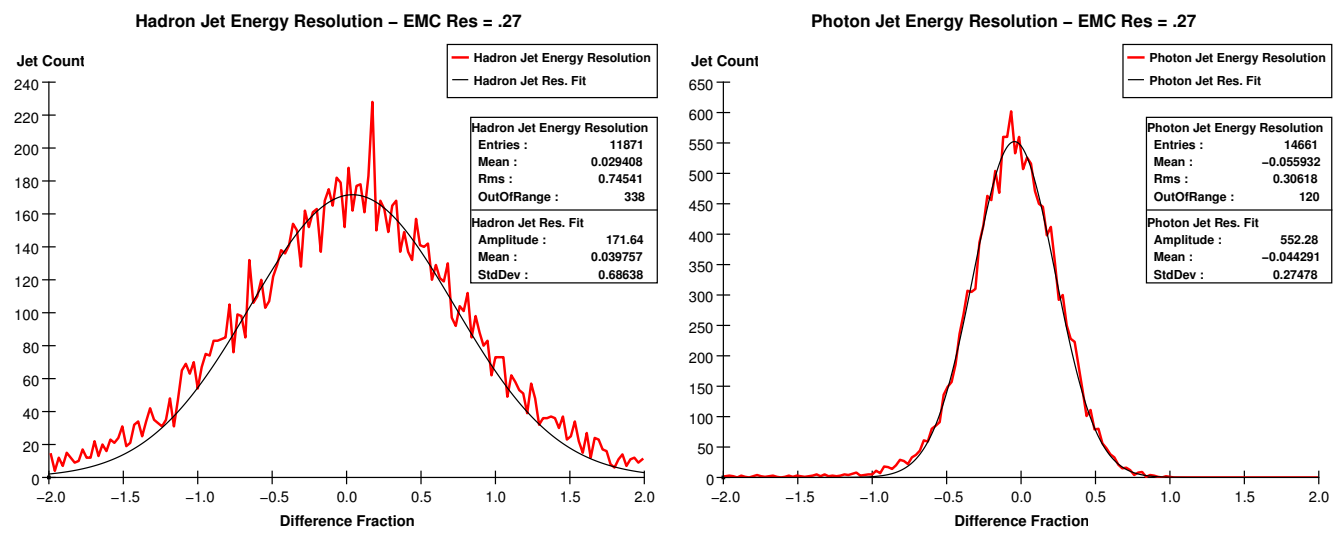

Figure 25: The above figure contains a plot of the photonic and the hadronic jet energy resolutions for the standard detector parameterization.
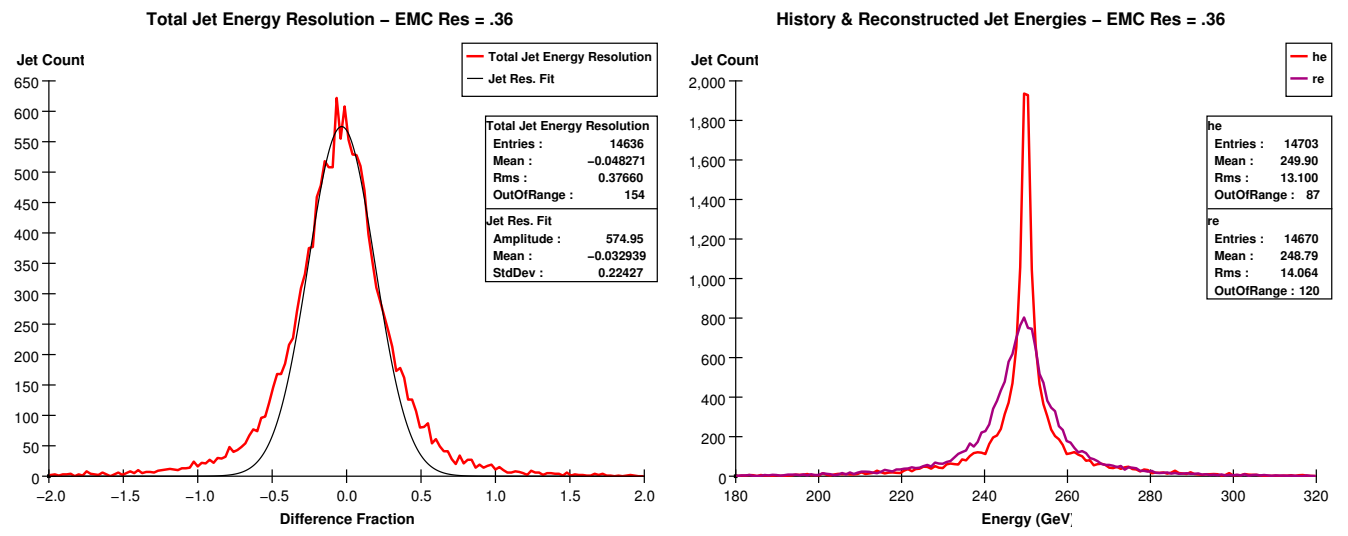

Figure 26: The above figure contains a plot of the photonic and the hadronic jet energy resolutions for the standard detector parameterization.

change observed between $a=.27$ and $a=.36$. This is a consequence of the important multicomponent nature of the total resolution; as the electromagnetic calorimeter gets blurrier, the sharpness of the peak depends more and more heavily on the other components of the detector, finally being carried completely by the tracker, which is the most accurate component. The effect of changing $a$ at high $a$ does still show itself, but now also as an increase 

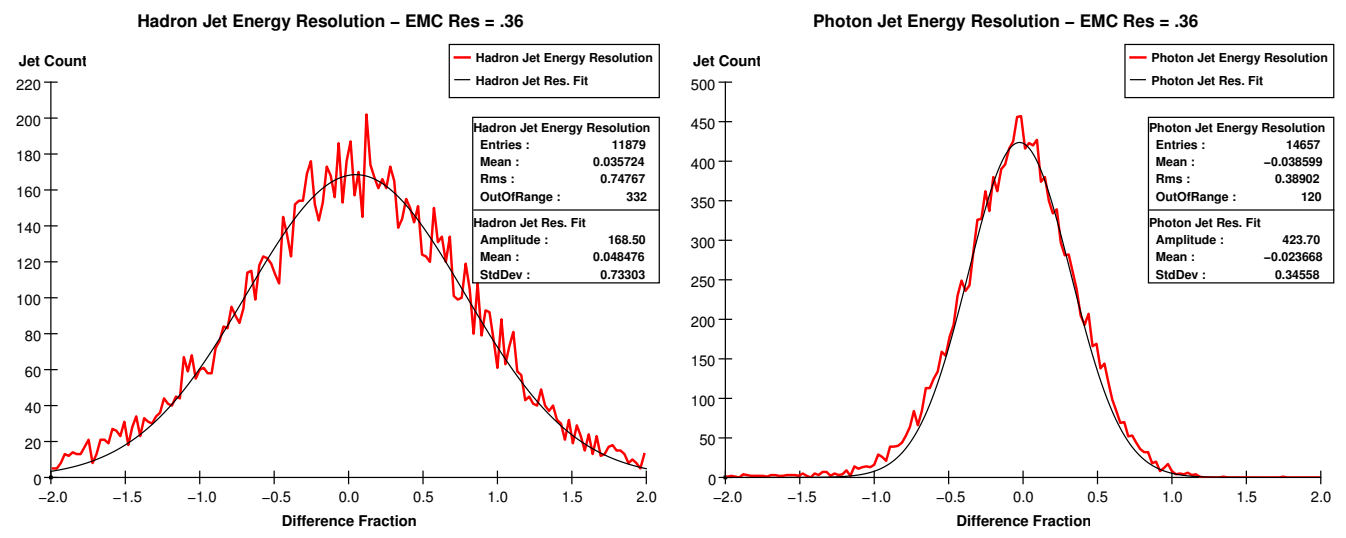

Figure 27: The above figure contains a plot of the photonic and the hadronic jet energy resolutions for the standard detector parameterization.

in the bulk of the "haunches" of the distribution, outside the core but not properly called part of the tail. Again, notice the effects of particle loss in the distributions. The most prominent Landau-esque non-hermeticity tail can be observed in the photon jet resolution plot for which $a=.09 ;^{3}$ this is a clear feature in this case mostly because the statistics are the best for this plot. However, we must be mindful that this effect is occurring in all histograms, and that this effect is responsible for the nonzero and negative means of the various distributions.

Now, let us turn our attention to the behavior of the resolution results as we change the hadronic calorimeter resolution. The plots below, namely Figures 28?33, contain again the total energy resolution, hadronic and photonic resolution, and raw energy peak for each parameter value we choose; values for the hadronic $a$ parameter in these figures include $a=.30$ and $a=.70$.

The effects of changing the hadronic resolution parameter $a$, though implemented almost identically in the program, come in sharp contrast to the behavior observed as we varied the electromagnetic parameter in Figures 22-27. Most prominently, the total jet energy resolution reaches a limiting

\footnotetext{
${ }^{3}$ see Figure 23
} 

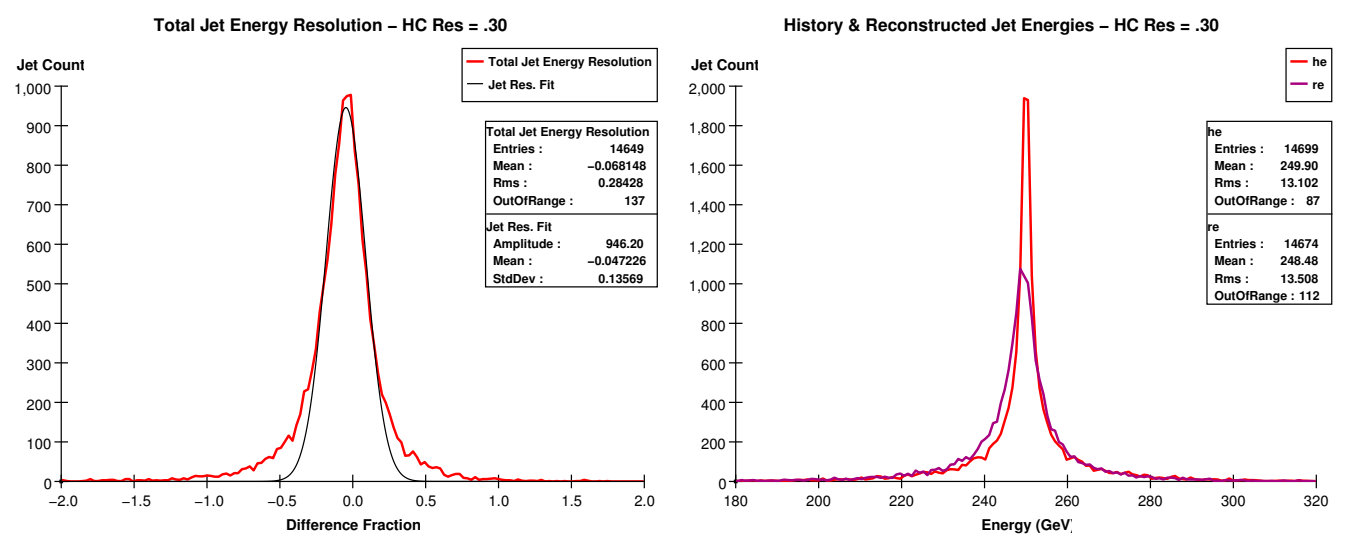

Figure 28: The above figure contains a plot of the photonic and the hadronic jet energy resolutions for the standard detector parameterization.
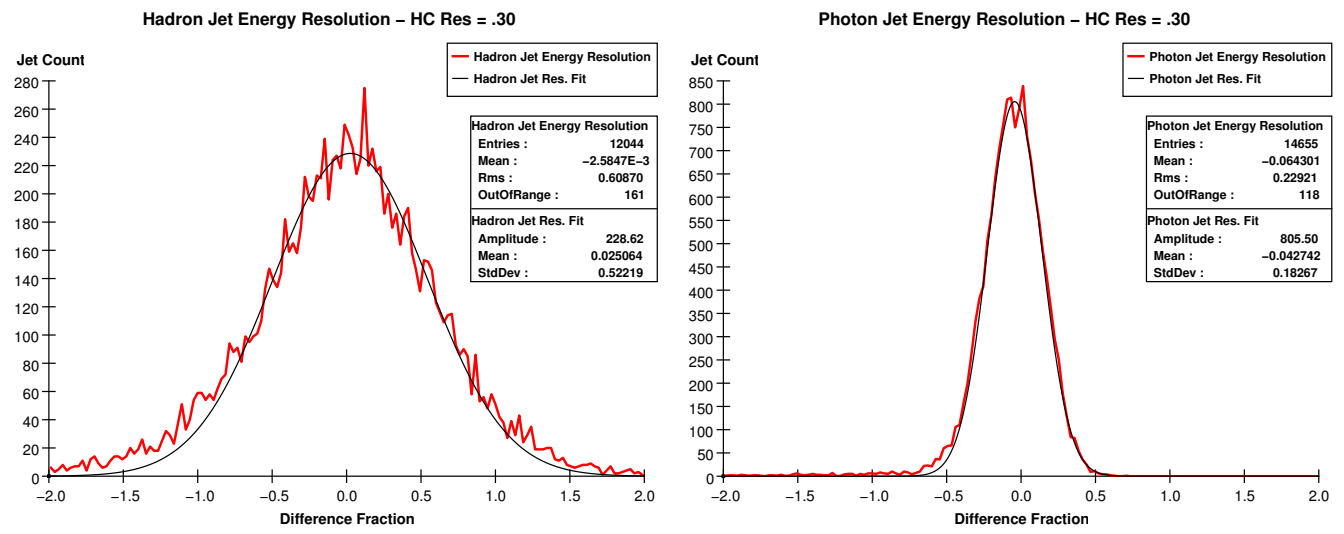

Figure 29: The above figure contains a plot of the photonic and the hadronic jet energy resolutions for the standard detector parameterization.

value much more rapidly than in the electromagnetic case, actually reversing direction by the time $a=.70$ : the sequence of total jet resolutions as $a$ is varied is, shockingly $13.569 \%, 15.177 \%$, and $15.033 \%$ ! The reversal of the trend requires the illumination of several points in order to become satisfactorily clear, the first of these points is the particle content in the particular

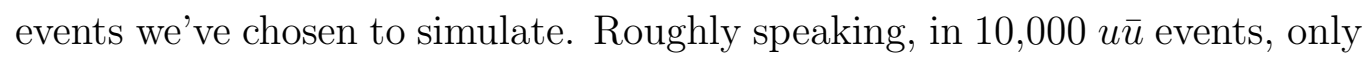



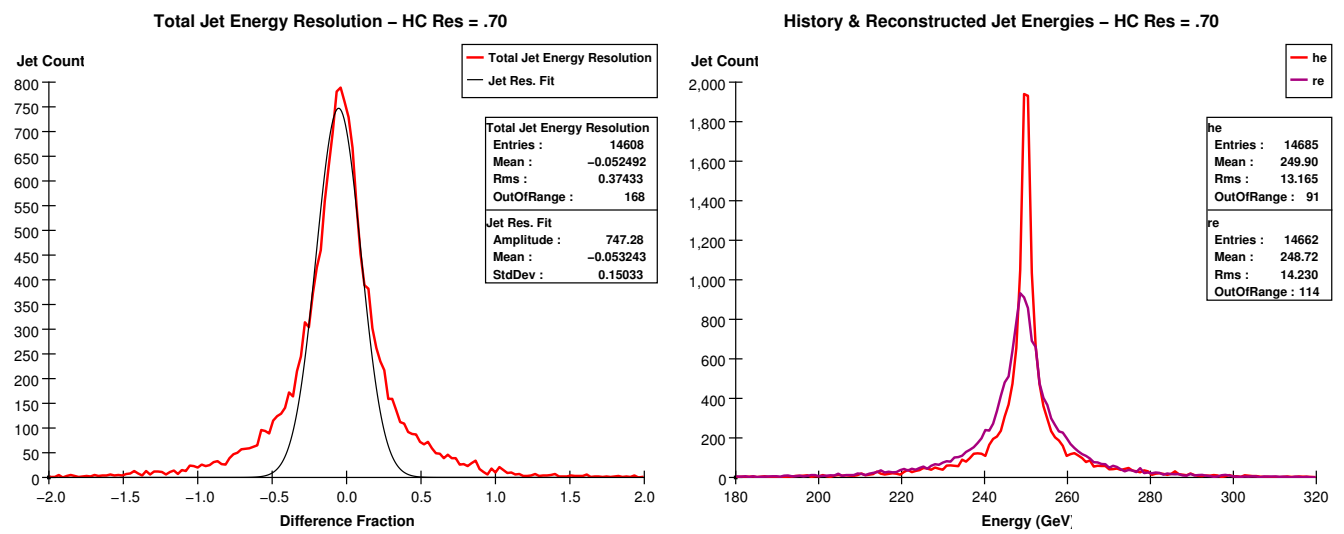

Figure 30: The above figure contains a plot of the photonic and the hadronic jet energy resolutions for the standard detector parameterization.
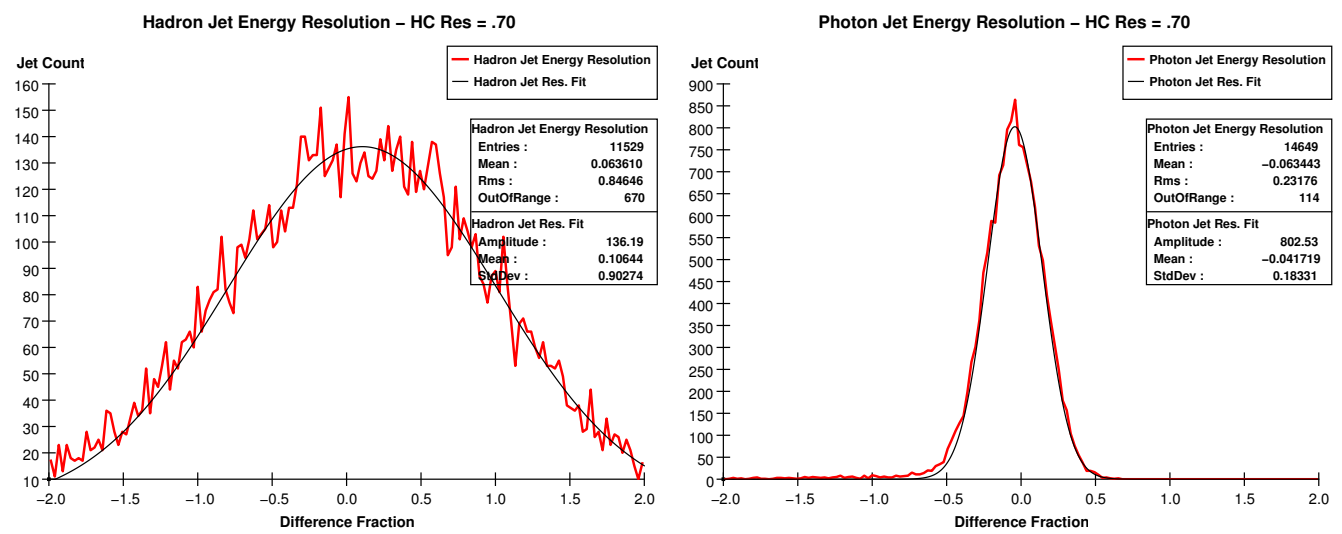

Figure 31: The above figure contains a plot of the photonic and the hadronic jet energy resolutions for the standard detector parameterization.

$5.3 \%$ of the particles produced are neutral hadrons, with the remainder about equally split between the charged particles and the photons. Consequently, we can reasonably expect that changing the hadronic calorimeter resolution will not affect the total jet resolution very much when compared with the effects of changing other components. However, an interesting thing occurs as we make the hadronic resolution very bad; The component of the total jet 
resolution due to hadrons becomes so spread out and consequently of such low amplitude that the core of the distribution to a good approximation is dominated by the photonic and charged components of the jets. Oddly, then, making the hadronic resolution very bad has the counterintuitive effect of actually improving the total jet resolution by effectively removing itself from the region of the distribution whose shape defines the resolution. As we would expect from the behavior we observed when the electromagnetic resolution was pushed up to .36, the "haunches" and tail region of the jet resolution histogram gain a great deal of bulk as the hadronic $a$ is set to $.70 .^{4}$

Let us lastly again turn our attention to effects incurred as a result of changing the tracker resolution parameters. Using again the parameterization set out in Equation 22, we generate Figures 32-39 found below by letting the parameters assume their "simple-standard" values", but with the a parameter varying between the values $1.0 \times 10^{-5}, 2.1 \times 10^{-5}, 4.0 \times 10^{-5}$, and $8.0 \times 10^{-5}$.
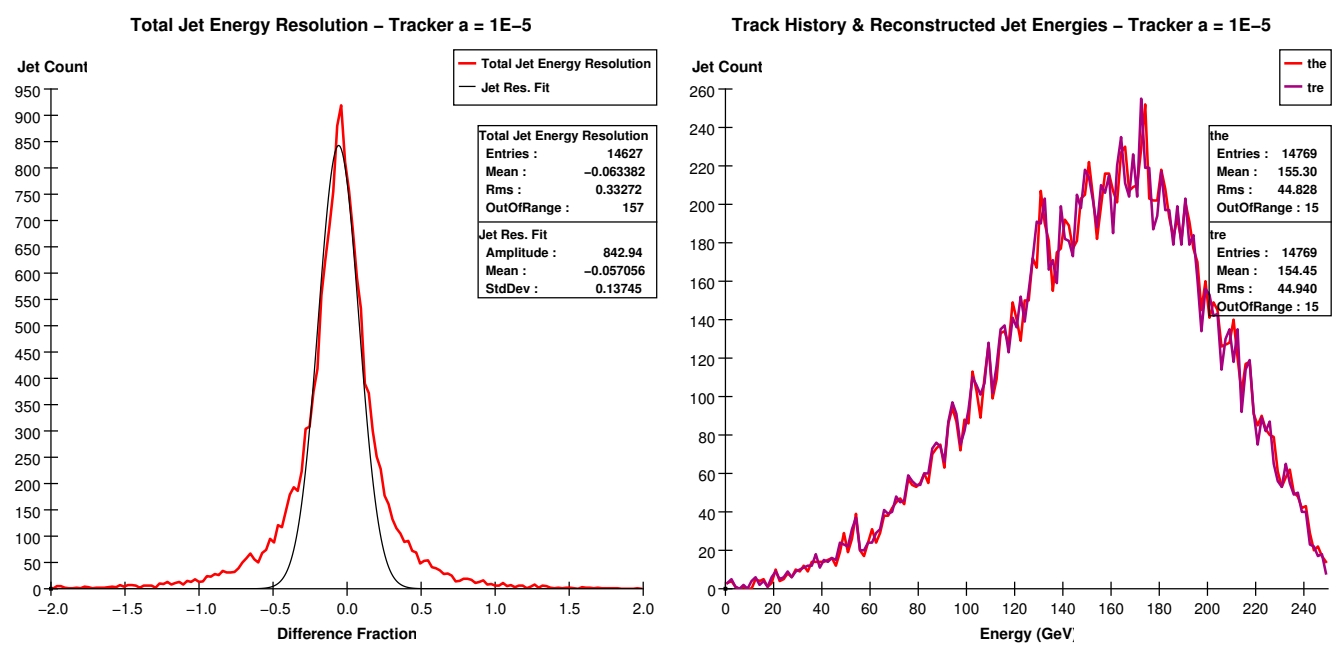

Figure 32: The above figure contains a plot of the photonic and the hadronic jet energy resolutions for the standard detector parameterization.

\footnotetext{
${ }^{4}$ see Figures 26 and 27.

5 these are defined on page XX.
} 

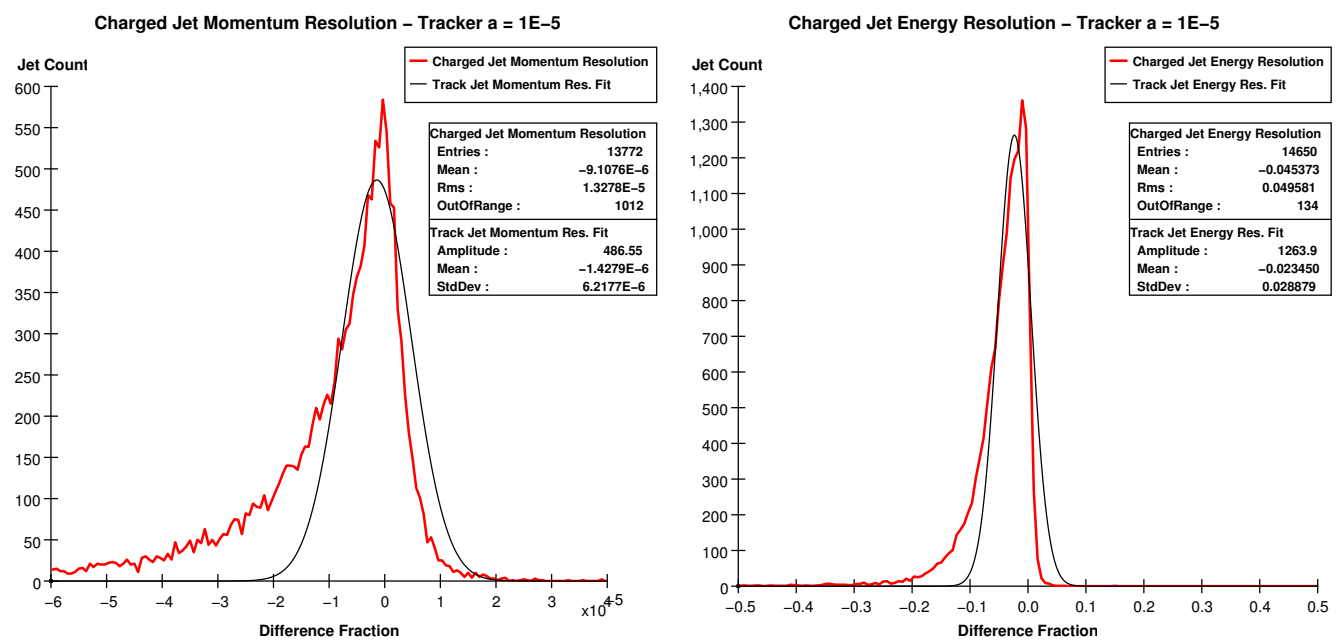

Figure 33: The above figure contains a plot of the photonic and the hadronic jet energy resolutions for the standard detector parameterization.

Right away, in the plots of the charged component of the jet resolution we can see that we are going to be severely handicapped by the tails that particle loss and inefficiency effects cause; this is especially true with respect to defining fits for the tracker resolution histograms. Also in Figure 32 we see the familiar "pointiness" at the mean of the jet resolution distribution that is the hallmark of a multivariate distribution that has a very sharply defined component. This feature will actually persist as we vary the tracker parameters, since the range of $a$ values we go through doesn't make the tracker less precise than the calorimeters.

After this first round of tracker examination histograms, we see that the effect of changing the tracker is, as expected, quite different from that of changing either of the calorimeters. Considering that we have two variables working synchronously to shape a single component of the complicated multivariate jet energy resolution, we should certainly be prepared to see some nontrivial dependences on the parameters that appear strange at first glance, requiring further explanation; thankfully, though, the behavior we observe 

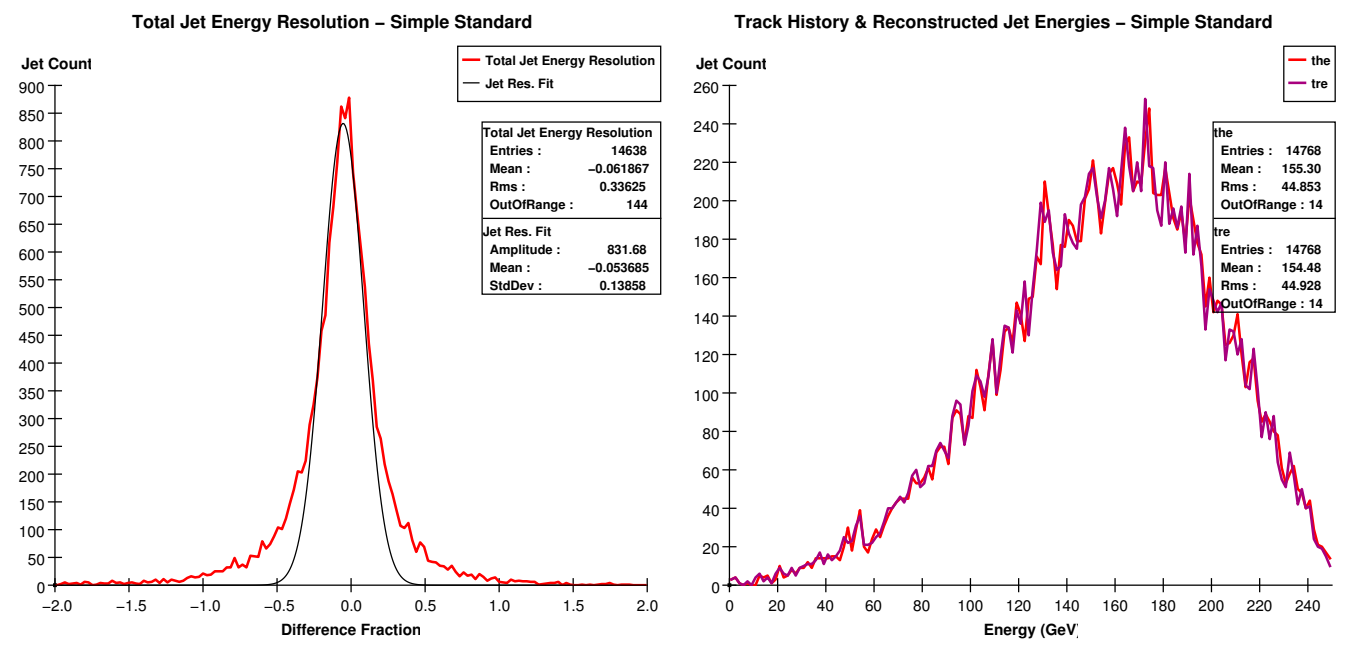

Figure 34: The above figure contains a plot of the photonic and the hadronic jet energy resolutions for the standard detector parameterization.
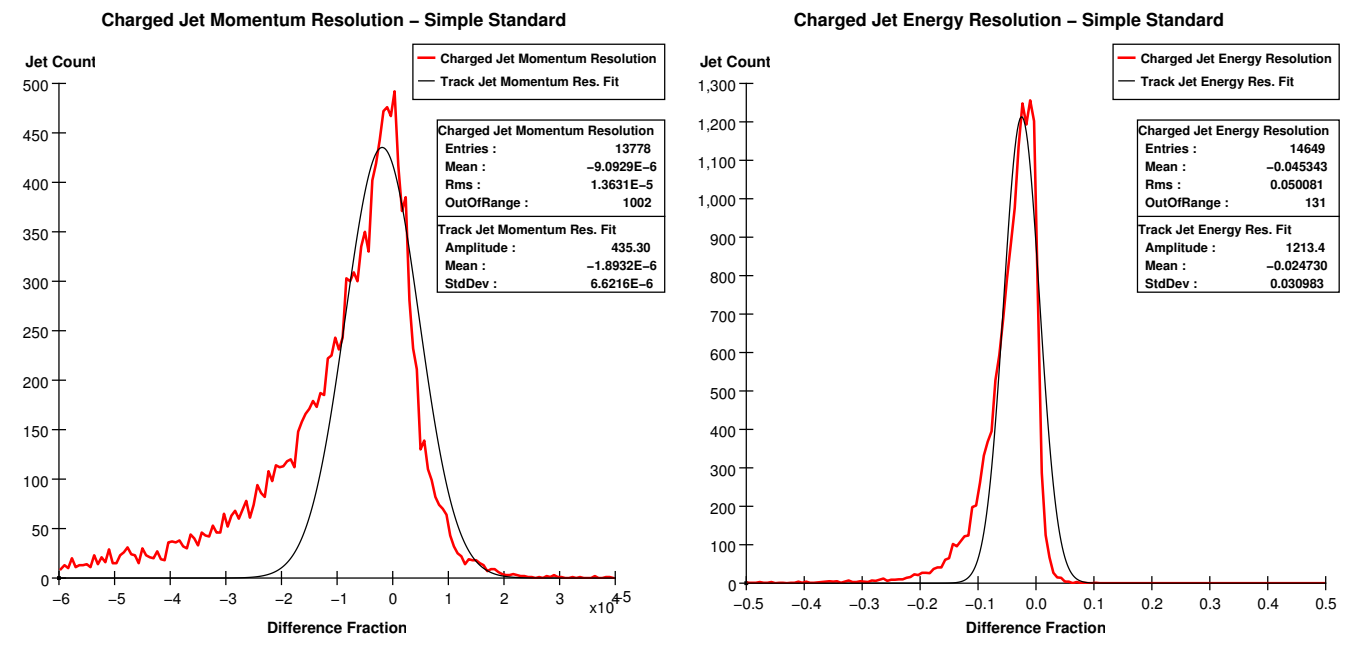

Figure 35: The above figure contains a plot of the photonic and the hadronic jet energy resolutions for the standard detector parameterization.

can be understood in the context of effects we have observed before (especially dependences seen on the hadronic calorimeter parameter). The jet energy resolutions we observe are, in order of increasing tracker $a: 13.745 \%$, 

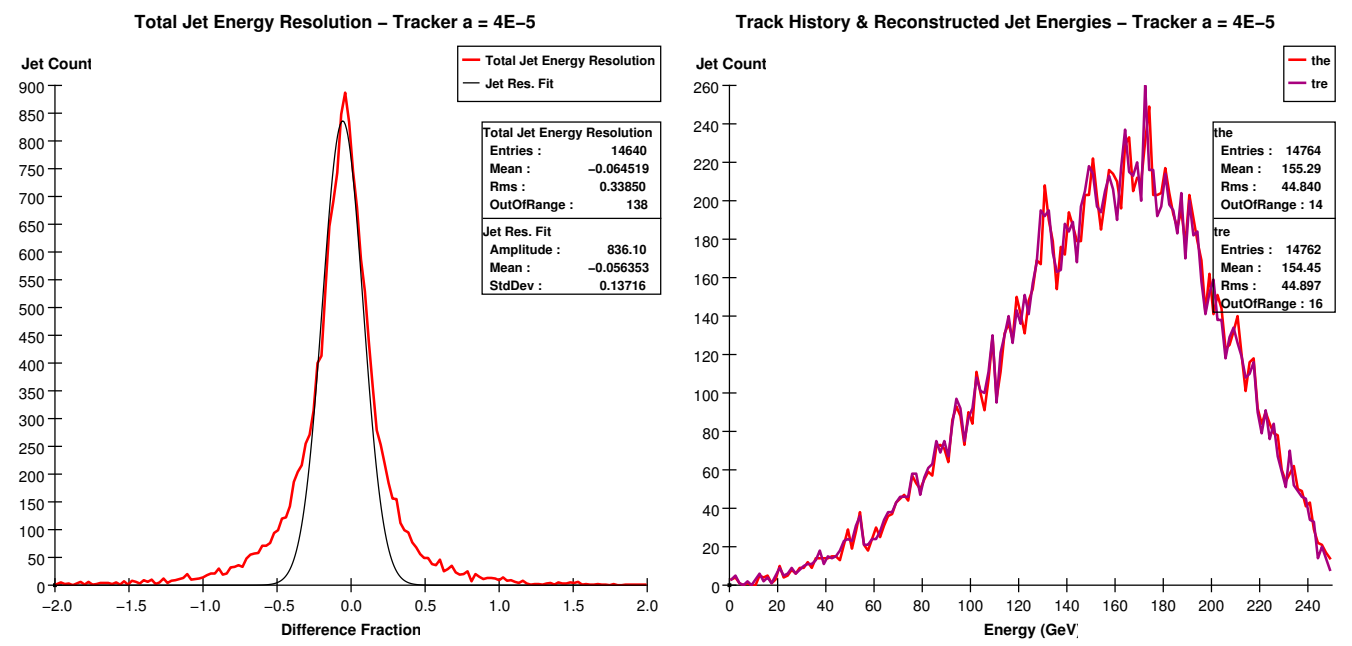

Figure 36: The above figure contains a plot of the photonic and the hadronic jet energy resolutions for the standard detector parameterization.
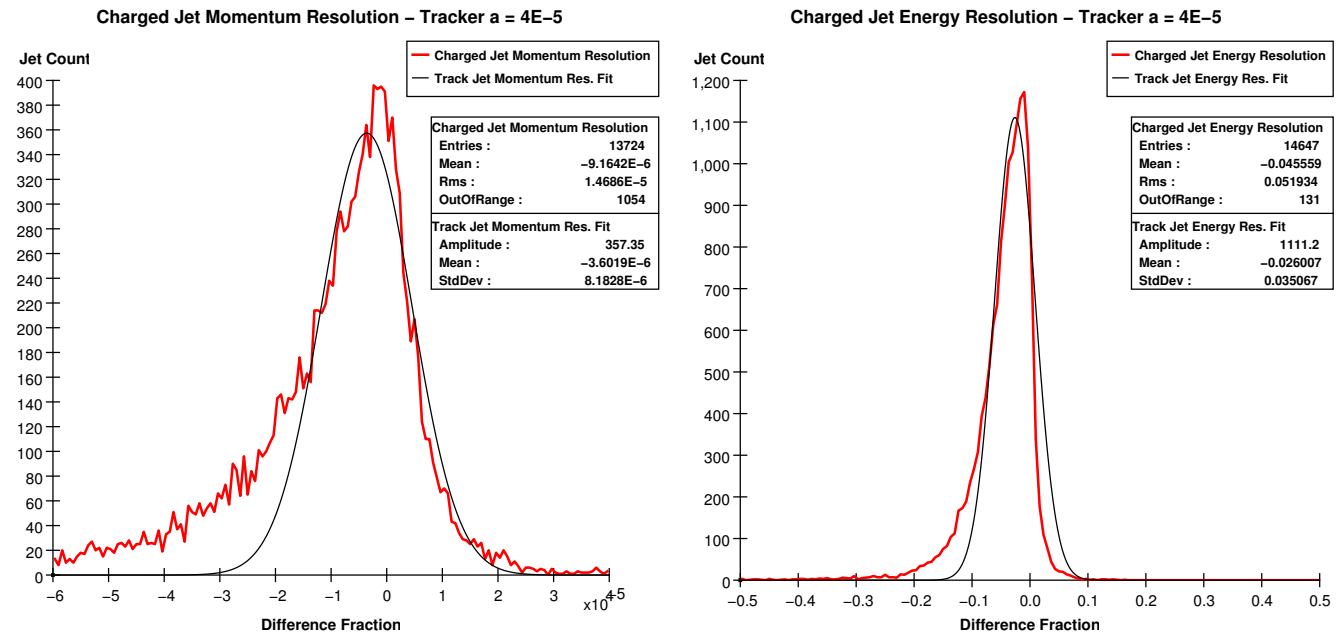

Figure 37: The above figure contains a plot of the photonic and the hadronic jet energy resolutions for the standard detector parameterization.

$13.858 \%, 13.716 \%$, and $15.014 \%$. Clearly this is a strange dependence, and we cannot just sweep it under the rug by calling it statistics since we have more than thirteen thousand jets going into each of these measurements. 

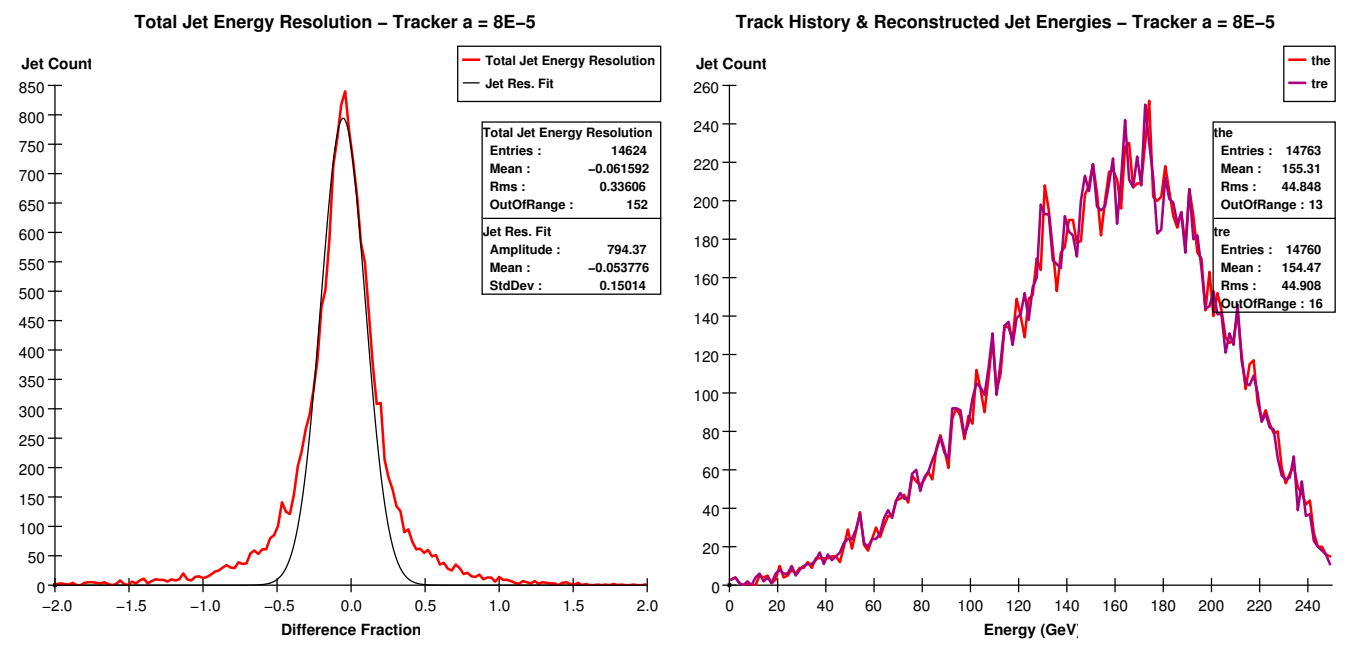

Figure 38: The above figure contains a plot of the photonic and the hadronic jet energy resolutions for the standard detector parameterization.
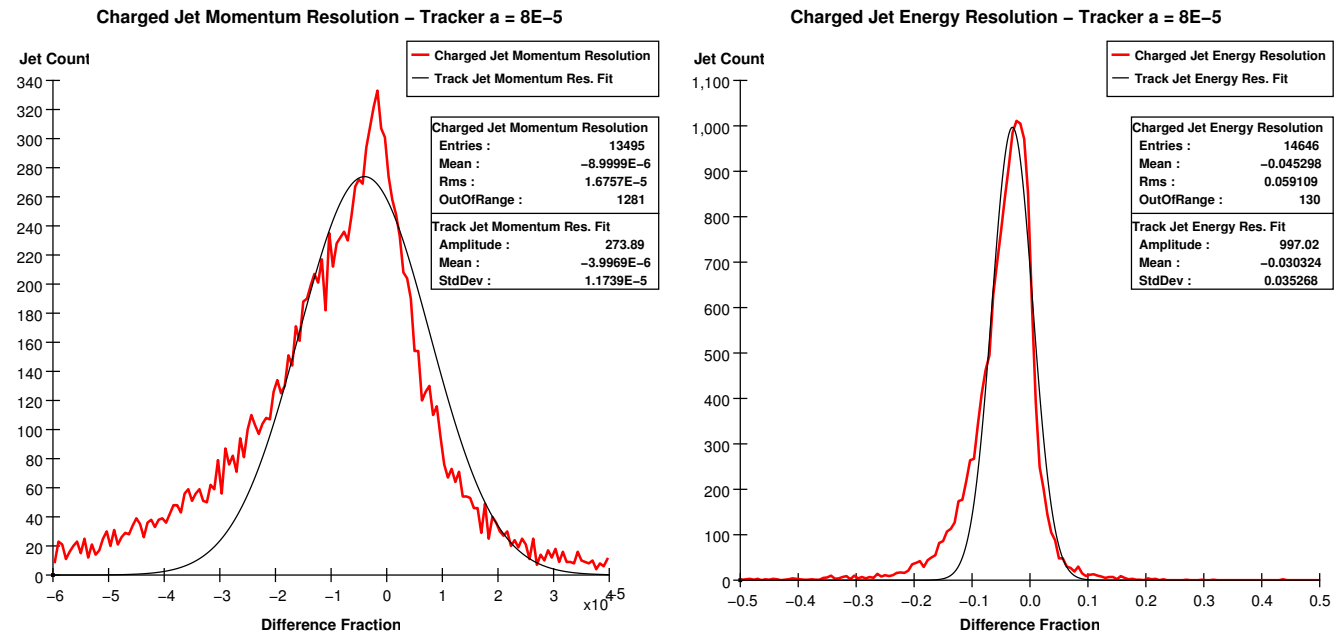

Figure 39: The above figure contains a plot of the photonic and the hadronic jet energy resolutions for the standard detector parameterization.

The key to this dependence is related to the sudden improvement observed in the jet energy resolution as the hadronic calorimeter parameter $a$ reached its upper limit: a situation in which we were looking at a distribution with 
several added components naturally falling into groups with quite different deviations. Whenever one examines a one dimensional distribution that is some kind of collapsing or projection of a distribution of two variables in which the deviation of thin, one dimensional cuts on the collapsed variable depends heavily on the actual position of the cut along the collapsed axis, the width of the core of the fully collapsed distribution will depend, to an excellent approximation, only on the parts of the original two dimensional distribution where the collapsed variable cuts had tight deviations, provided the dependence on the collapsed variable isn't so great as to make this sharp region negligibly small. We can put this important general statement into context by looking at the charged component of the jet energy resolution in this way; the distribution for the charged jet energy resolution is, as Equation 22 suggests, a distribution in two variables: the energy $E$ and the angle theta. The degree to which the distribution depends on theta is given by tracker parameter $b$, so in light of the above statement, if we have a large $b$, the resulting charged jet energy resolution should have a core that is really only a picture of the regions in theta where the jet energy resolution is good. However, if we make $a$ (not $b$ !) large enough that we can say $a \gg b$, then the well-defined $\theta$ dependence on which the selection phenomenon depends will be swamped out. Also, we expect that if we make $b$ very large we can cause a similar effect, only this time the resulting distribution will have such a small region in theta for which the resolution is good that a projection onto the energy axis will not have a well-defined core. So, as we change parameters, we expect four distinct regimes: if the resolution is just plain good enough, the core will be very tight; as the resolution gets worse but not too bad, the variations along the collapsed axis serve to smear this resolution around further, without "selecting" a particular region to define a core; as we make the resolution yet worse the selection phenomenon occurs, causing a sharpening of the core, as observed when the hadronic calorimeter parameter $a$ reached its highest value; finally we expect as the resolution gets yet worse, the selec- 
tion will be on such a small region that the collapsed distribution's core will lose out to the enormous, encroaching haunches that the heavy dependence causes. We can see an example of the selection phenomenon occurring in Figures 33, 35, 37, and 39 in the rightmost histogram. However, since our total jet energy resolution is a composite distribution of a few one dimensional distributions (the calorimeters) anda collapsed two dimensional distribution (the tracker) we expect end behavior in the extreme fourth regime where the tracker resolution gets so bad that the total jet energy resolution's core becomes almost totally photonic. All of this, to complicate things even more, occurs in the presence of a debilitating Landau-type particle loss tail in the tracker that skews everything, weakening any quantitative arguments based on gaussian fits. Having finally dealt fully with the complex statistical concepts necessary for understanding these collapsed distributions, we can turn our attention to $b$-parameter dependence, Figures 40-45.
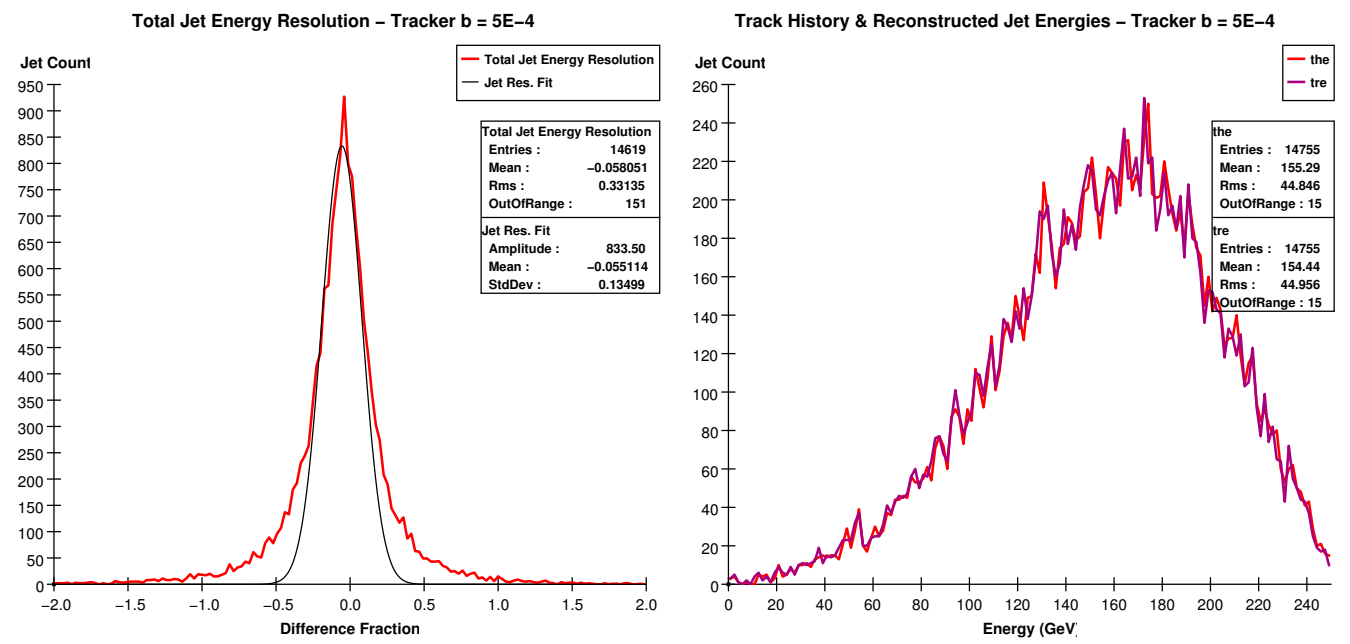

Figure 40: The above figure contains a plot of the photonic and the hadronic jet energy resolutions for the standard detector parameterization.

Note that in the sequence of Figures 40-45 the Simple Standard Figures 34 and 35, for which $b=1 \times 10^{-3}$, should come between Figures 41 and 42 

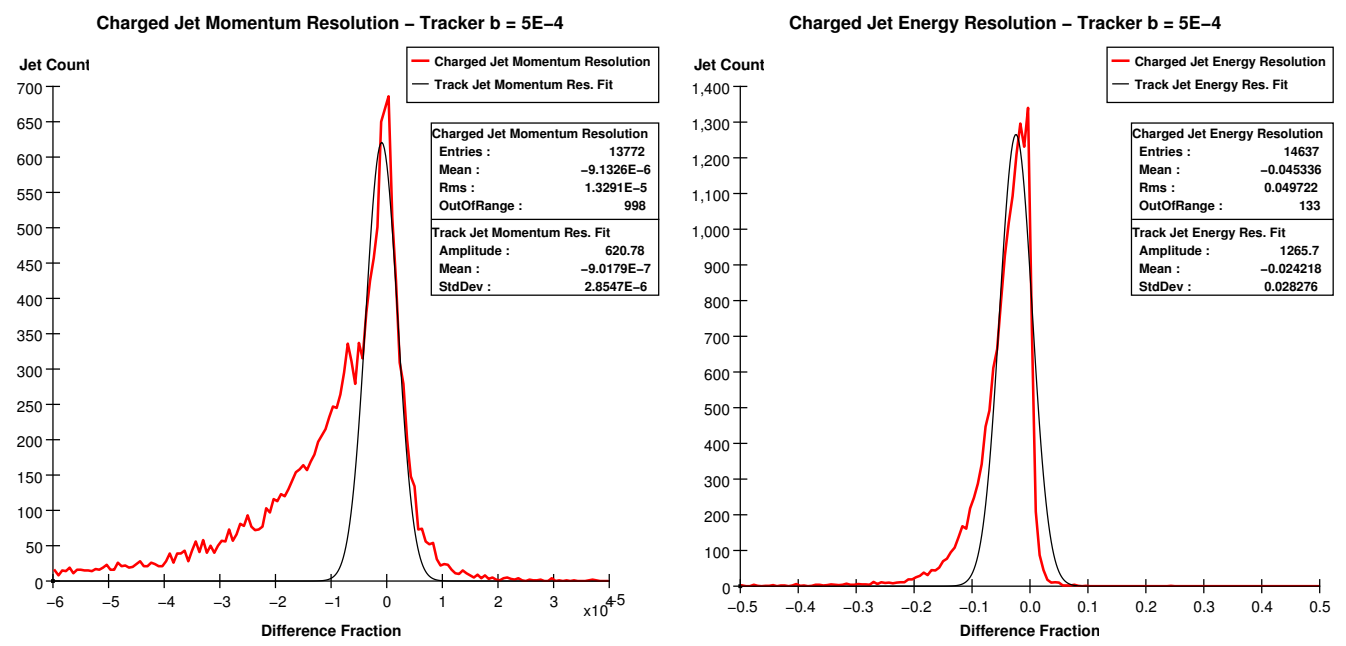

Figure 41: The above figure contains a plot of the photonic and the hadronic jet energy resolutions for the standard detector parameterization.
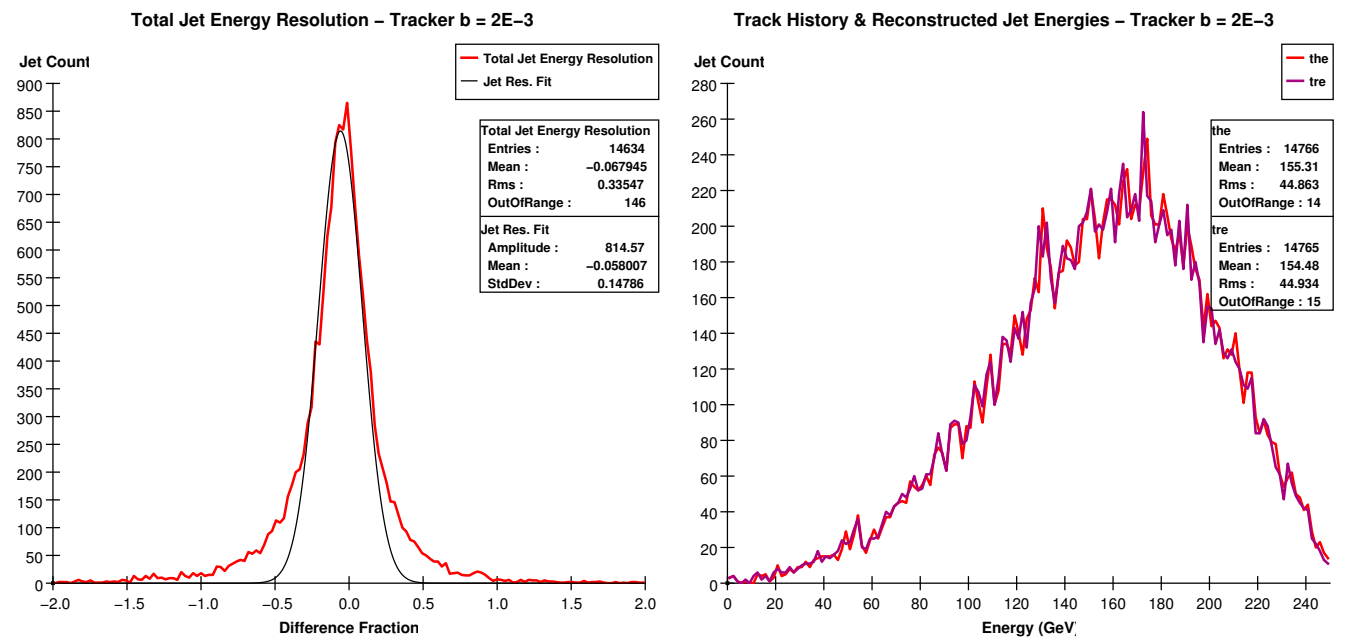

Figure 42: The above figure contains a plot of the photonic and the hadronic jet energy resolutions for the standard detector parameterization.

in order to complete the sequence. Just as in the histograms where the $a$ parameter was varied, we see the "selection?? process occurring in the total jet energy resolution plots (the left histogram in the even numbered figures). 

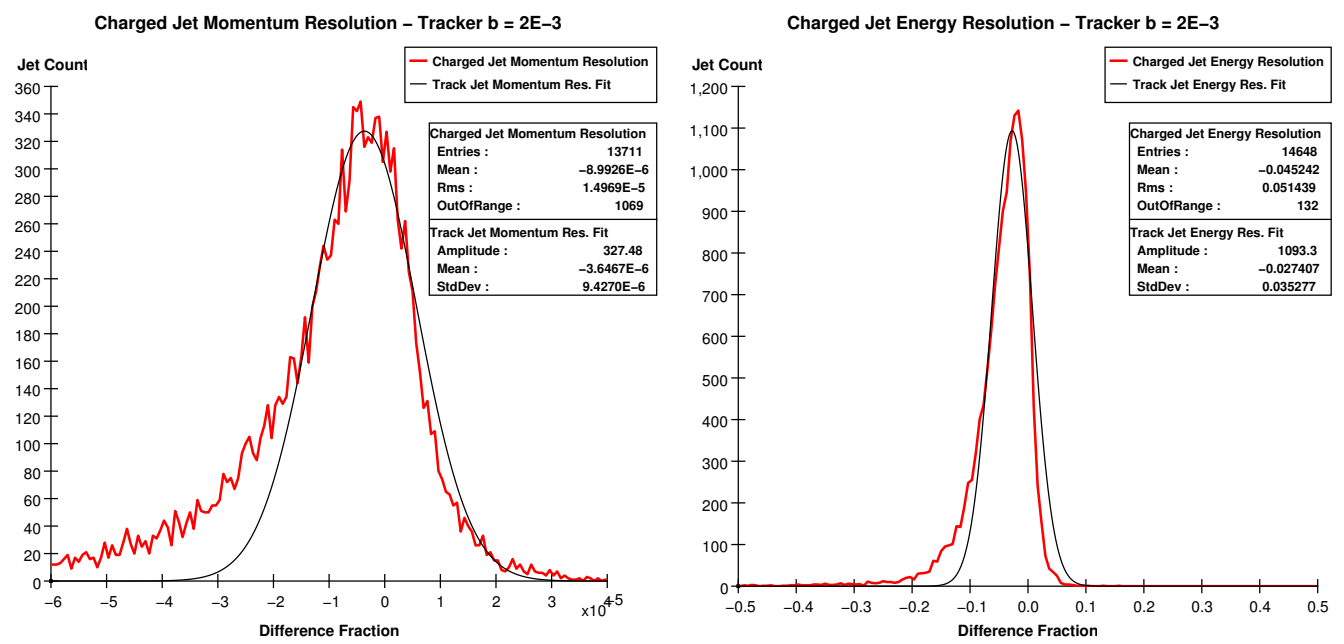

Figure 43: The above figure contains a plot of the photonic and the hadronic jet energy resolutions for the standard detector parameterization.
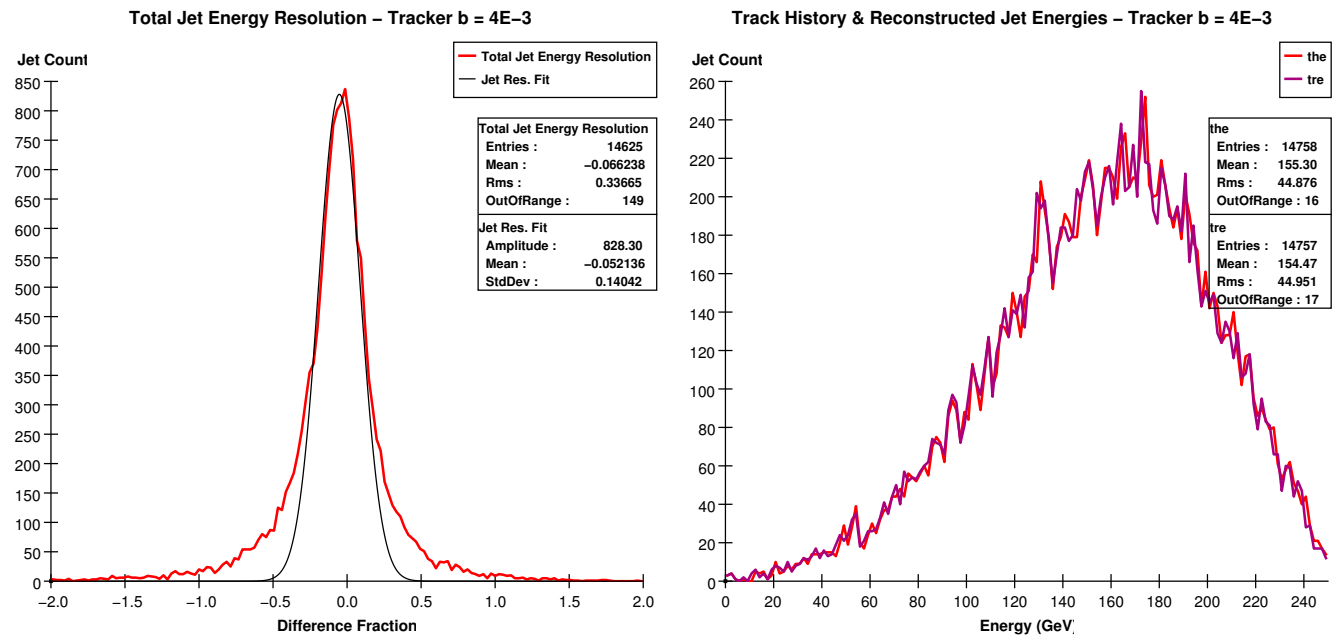

Figure 44: The above figure contains a plot of the photonic and the hadronic jet energy resolutions for the standard detector parameterization.

Interestingly though, in this set of histograms we don?t see the selection phenomenon losing out to poor resolution for high values of $b$, something we must account for. By looking at Equation 22, we see that near $\theta=0$, Pt will 

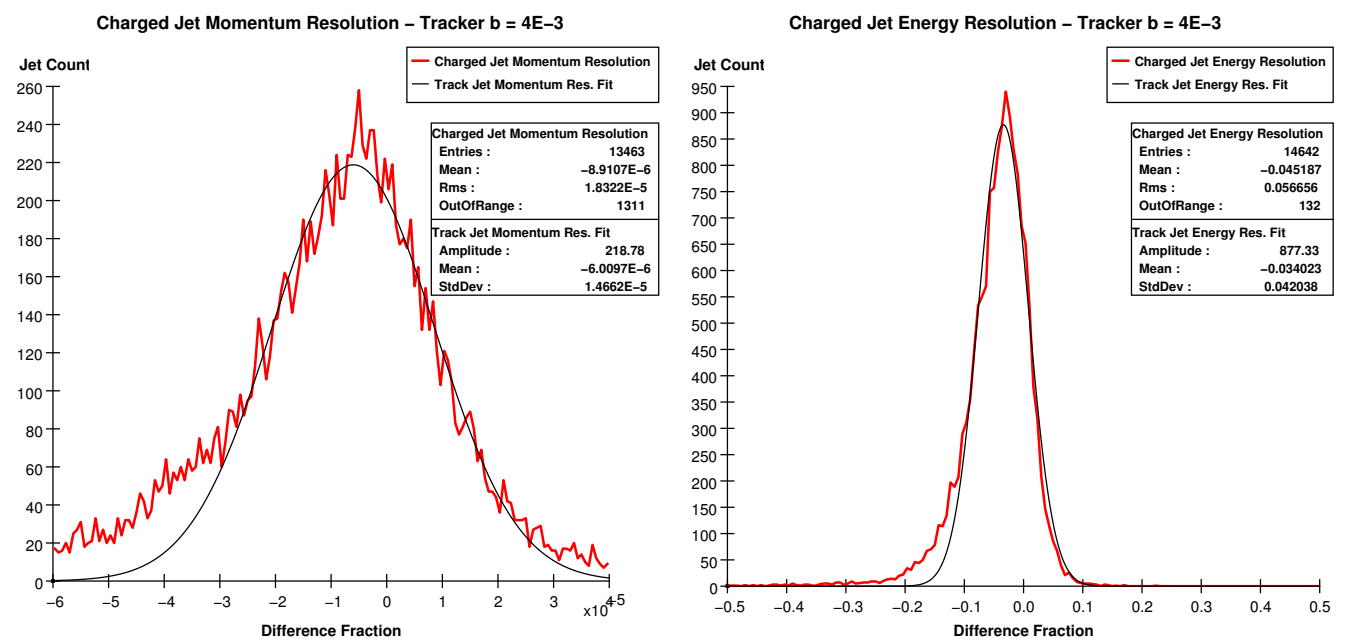

Figure 45: The above figure contains a plot of the photonic and the hadronic jet energy resolutions for the standard detector parameterization.

be maximized for a particle of some arbitrary energy, thus minimizing the contribution from the $b$ parameter over a range of angles centered around $\theta=0$. Parameter $a$, being isolated as a constant term, exhibits no such behavior; making a large will after a certain point affect all particles equally. The fact that we have particles selecting to minimize their errors if they come in at small angles and reasonably high energies will cause the selection phenomenon to persist over a much greater range of $b$ values than $a$ values. This is in agreement with the statistical exposition above; we cite $a$ and $b$ as affecting the total distribution in different ways, and even though the final effects are similar we shouldn't expect the different regimes to partition the $a$ and $b$ continuums identically. That being said, if we increased $b$ enough, we should see the resolution trend inverting and getting worse, then finally settling on whatever value the photonic calorimeter and hadronic calorimeter can support. A final note, we can see that there are less and less counts in the core of the total jet energy distribution by looking at the peak values at the centers of the plots, supporting our ideas behind the cause of the selection 
phenomenon.

\section{$5 \quad$ Physics Analysis}

Finally, after having put the program through extensive analysis and testing, we will use it to perform a somewhat more realistic analysis; in this section we present plots and results from analyzing a T-channel process in which an electron positron pair produce a pair of $\mathrm{Z}_{0}$ bosons. The lowest-order Feynman diagram for this process appears below, in Figure 46. Our particular simulated event takes place at $500 \mathrm{GeV}$ center-of-mass energy, making the results appropriate for a thrust analysis as the jets from the $Z_{0}$ particles will have good separation at this energy. In order to "measure" the masses of the $\mathrm{Z}_{0}$ bosons generated, we simply use the thrust axis of the event to divide the detector up into thrust hemispheres, then sum up the measured four-vectors of the final-state jet particles over each hemisphere. The end result will, of course, be two composite four vectors for each jet; we simply call the measured masses of these composite vectors the measured mass of the $\mathrm{Z}_{0}$ particle which creates the jet. Below, we present histograms of these measured $Z_{0}$ masses generated at various detector parameters (we use the same set from the previous section), along with a rudimentary analysis of the results we have obtained.

In this section of the paper, we shall choose not to undergo the exhaustive and laborious analysis of each detector component undertaken in the sections dealing with the $u \bar{u}$-type events. Instead, we will simply note the similarities in statistical changes for the histograms as we vary a detector parameter, letting the exhaustiveness of the former treatment argue that the causes must be the same, as these two-jet events are quite similar from the detector?s point of view. If anything, the fact that the pair of $\mathrm{Z}_{0}$ bosons has a much higher mass than the $u \bar{u}$ pair will only slightly affect the validity of our choice to treat these events with a thrust analysis. Again, the purpose of this section 


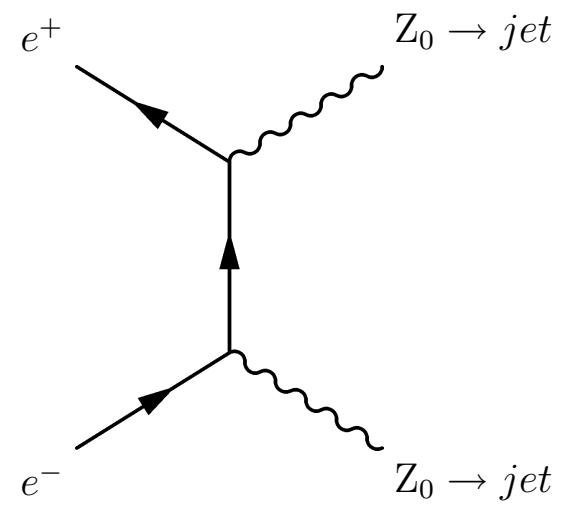

Figure 46: The above diagram depicts the T-channel $e^{+} e^{-} \rightarrow Z_{0} Z_{0}$ process simulated to create the figures in this section.

of the paper is just to show that the program can similarly handle a slightly more sophisticated event.
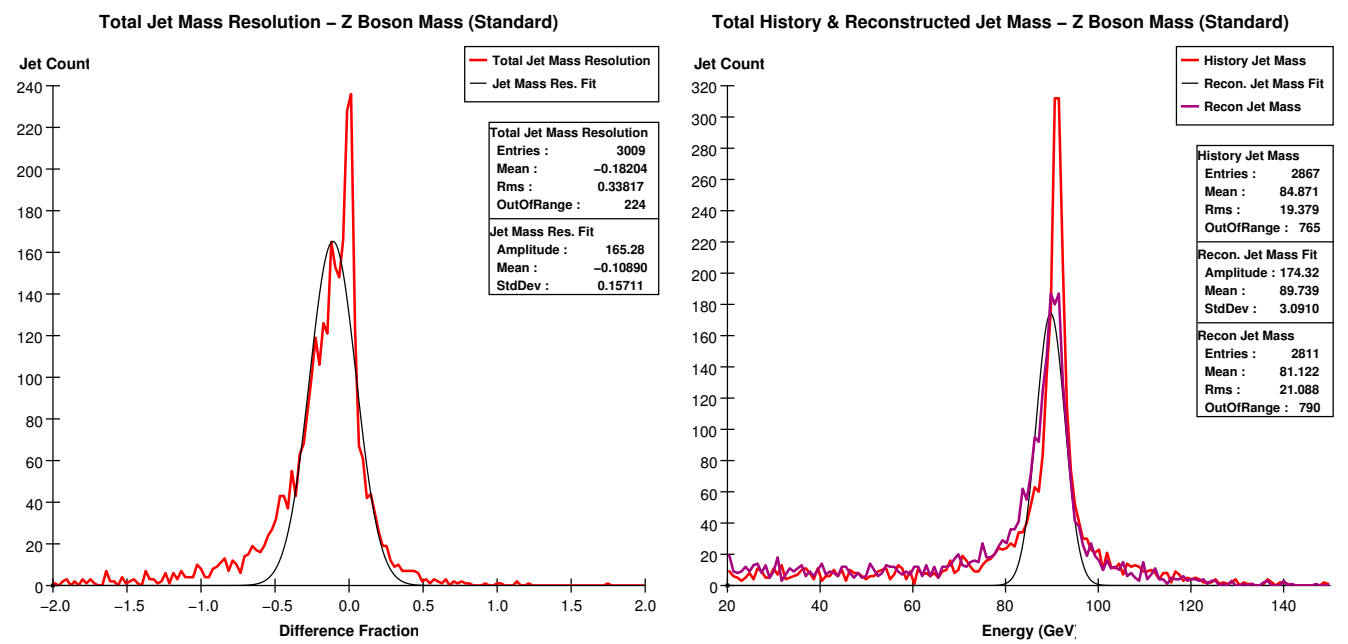

Figure 47: The above figure contains a plot of the photonic and the hadronic jet energy resolutions for the standard detector parameterization.

With the purpose of simple exposition in mind, we present firstly in Figure 47 two histograms summarizing the results of the $e^{+} e^{-} \rightarrow \mathrm{Z}_{0} \mathrm{Z}_{0}$ event simulated with standard detector parameters. Comparing this diagram with its 
$u \bar{u}$ analogue, Figure 16, proves especially useful. Both simulations, using the standard detector parameters, are subject to particle loss in the beampipe, the calorimeter because of minimum energy requirements, and the tracker because of minimum transverse momentum requirements; these losses manifest themselves in the negative shift of the reconstructed jet mass peak as compared with the history jet mass peak. Our standard detector reports a $\mathrm{Z}_{0}$ boson mass of $89.739 \mathrm{GeV}$, as compared with the modern measured value of $91.2 \mathrm{GeV}$. This does not seem spectacular, but the reader will notice that the center of the Gaussian fit in Figure 47 is certainly a bit below the center of the reconstructed mass peak, pushing our actual measured value a bit up and hence into a bit better agreement. This discrepancy is entirely due to the definition of the core of the peak, which is arbitrarily chosen to be all the distribution that falls above half the maximum height. This type of definition for the core is, unfortunately, necessarily somewhat blunt with the simple QR decomposition we've chosen to use. However, considering the complexity of the peak to which we must fit and the simplicity of the QR fitter, a disagreement of $1.6 \%$ should really be interpreted as very reassuring.

The next figure we present, Figure 48, summarizes a simulation for which the detector was hermetically sealed. Again, we can compare to the corresponding $u \bar{u}$ histograms, found in Figure 19 for some useful conclusions. As compared with their standard detector counterparts, both of these histograms exhibit much better agreement between the reconstructed mass/energy peaks and the history mass/energy peaks. We conclude, then, that in the simulation of the two $Z_{0}$ jets, the somewhat anemic mass measured with the standard detector parameters is due to particle loss. Confirming this hypothesis is the measured $\mathrm{Z}_{0}$ boson mass, reported in the hermetically sealed detector at $90.5 \mathrm{GeV}$. Again the reader can visually determine that the fit is again just a tiny bit shy of the position of the actual peak. Note here in comparison with Figure 47 that the peak for the hermetic detector is just a bit tighter than that for the standard detector; this is another trend that carries over 

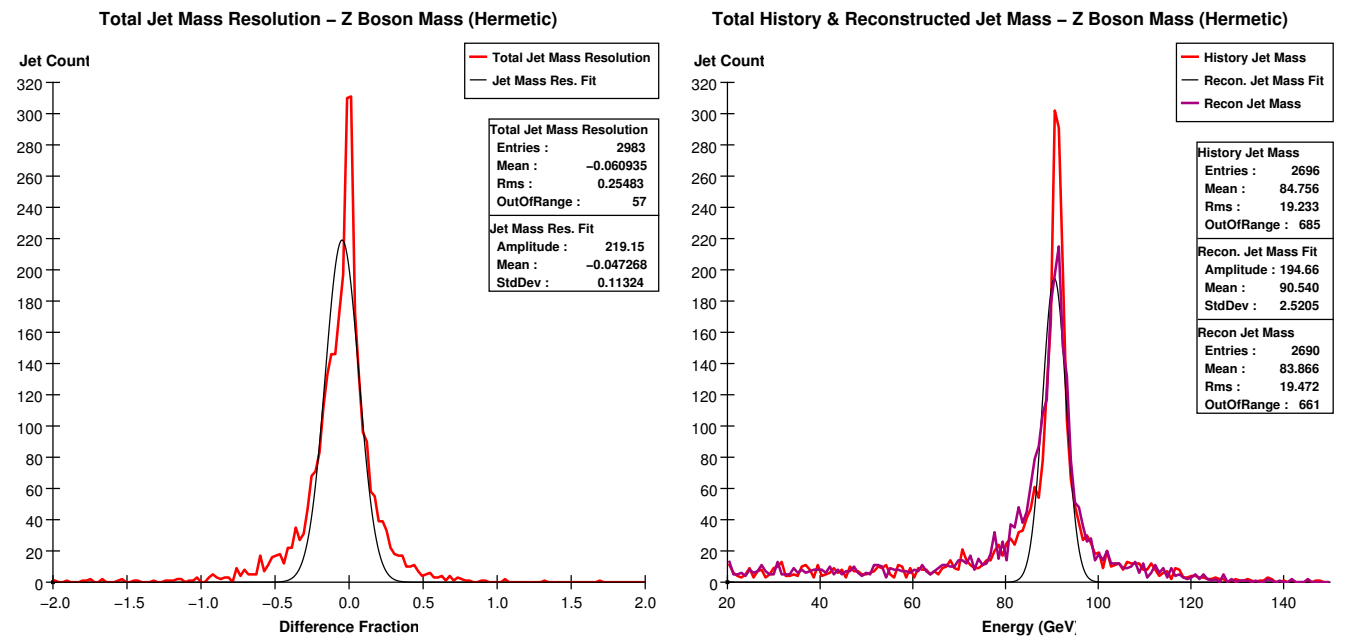

Figure 48: The above figure contains a plot of the photonic and the hadronic jet energy resolutions for the standard detector parameterization.

from the $u \bar{u}$ simulations.
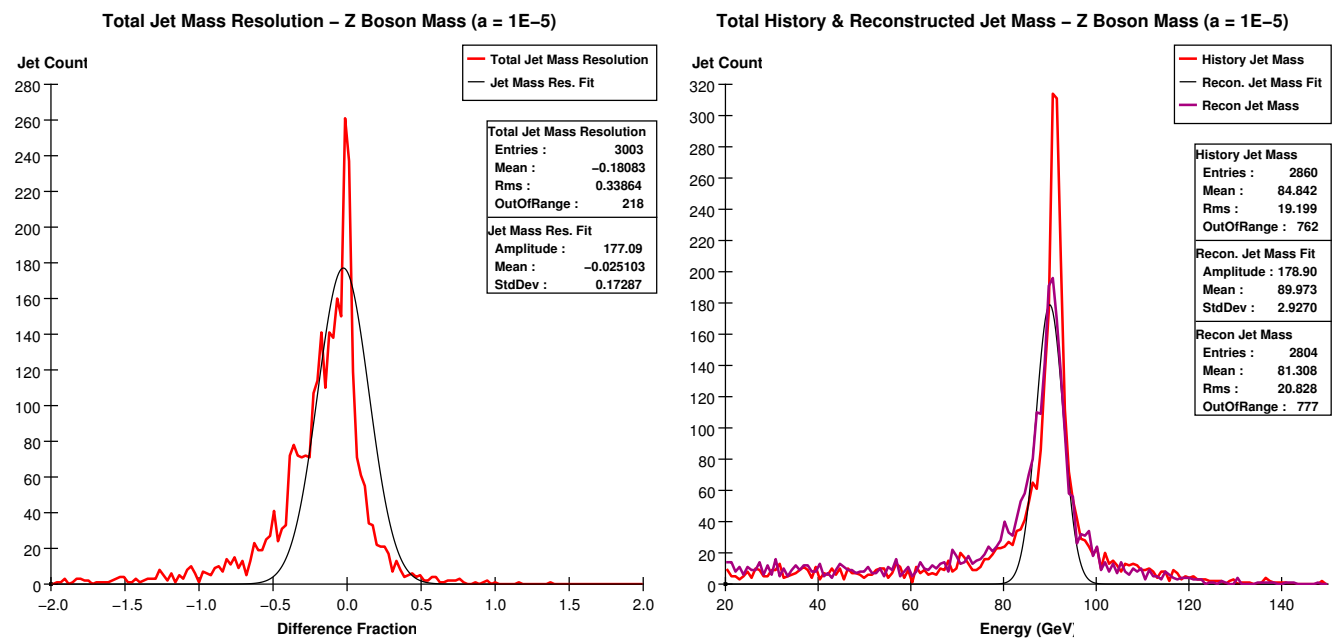

Figure 49: The above figure contains a plot of the photonic and the hadronic jet energy resolutions for the standard detector parameterization.

For the final point in the paper, we shall simply present a series of three 

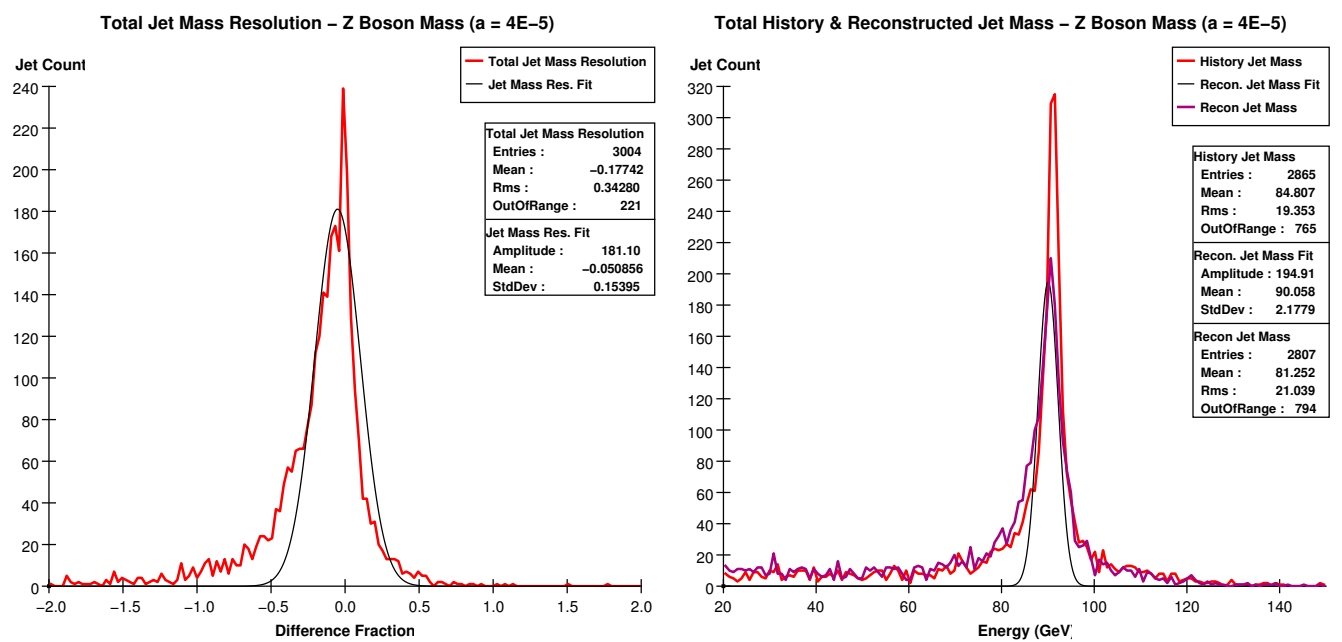

Figure 50: The above figure contains a plot of the photonic and the hadronic jet energy resolutions for the standard detector parameterization.
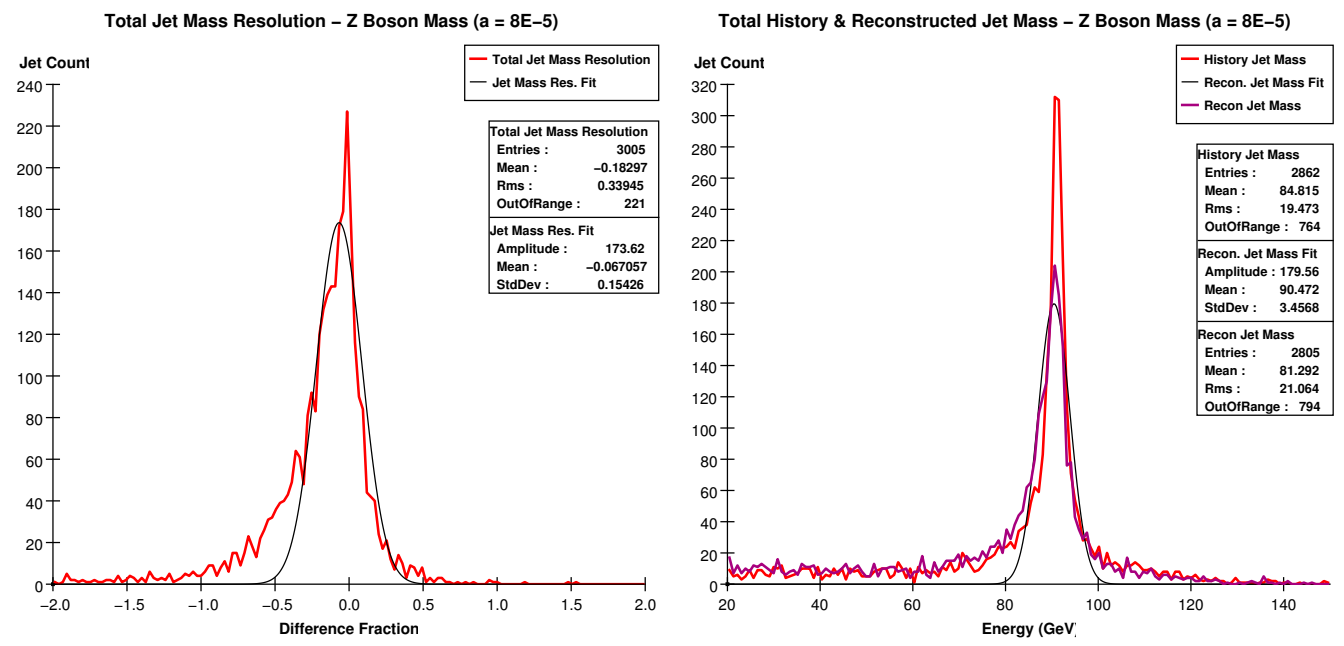

Figure 51: The above figure contains a plot of the photonic and the hadronic jet energy resolutions for the standard detector parameterization.

histograms generated over a range for a single detector parameter. The final histograms, found in Figures 49-51, are generated using the alternative simple tracker parameterization, with the tracker parameter $a$ as given in Equation 
22 acquiring the values $1 \times 10^{-5}, 4 \times 10^{-5}$ and $8 \times 10^{-5}$, respectively. Recalling the complex discussion about core selection in multivariate statistical distributions given previously in the paper, we should ostensibly be looking for the same features occurring as we vary a parameter in this simulation. However, upon inspection of the histograms, it is immediately apparent that our tails due to particle loss are much too large and our distributions are hence much too non-Gaussian to make any arguments that could be called compelling. Still, we should look for something familiar happening underneath the noise. Firstly, consider the left side Figure 40. This histogram was created by binning jet-by-jet the difference between the reconstructed and the history masses and then scaling appropriately; this is exactly the quantity binned in generating the jet energy resolutions in the $u \bar{u}$ sections of the paper. First of all, we see a very sharp peak that is clearly centered at 0.0 or somewhere very close to this value. Then, a third of the way down the peak, we see a large mass on the low energy side that is reminiscent of the tail/haunches incurred due to particle losses in the left side of Figure 33. In both of these histograms (each generated using the simple tracker parameterization with $a=1 \times 10^{-5}$ ) we have a sharply defined core due to the accuracy of the tracker along with the particle losses, and on top of this we have some selection occurring that sharpens the distribution if $\theta$ is close to 0 . A difference here is that we see the total jet mass resolution in a $e^{+} e^{-} \rightarrow \mathrm{Z}_{0} \mathrm{Z}_{0}$ event resembling the charged momentum resolution in a $e^{+} e^{-} \rightarrow u \bar{u}$ event; this fact suggests to us that we have a much greater charged component in the $e^{+} e^{-} \rightarrow \mathrm{Z}_{0} \mathrm{Z}_{0}$ type jets as compared to the $e^{+} e^{-} \rightarrow u \bar{u}$ type jets, which of course means that we are really going to be disabled in our analysis by the heavy particle losses in the tracker. Just as expected, for the histograms with the higher values for the $a$ parameter, we see the resolutions worsening. The main manifestation of this is in the core of the total jet mass resolution; as $a$ is increased, we see the core spike widening and dropping-by $a=4 \times 10^{-5}$ we already see the core at about the same height as the particle loss tail, but 
then as we move to double this value at $a=8 \times 10^{-5}$, there isn?t a huge difference in the look of the mass resolution plot. This signals us just as in the $u \bar{u}$ events that we have hit a transitional point where the accuracy of the other components of the detector are beginning to hold up the accuracy of the measurements to a greater degree.

This concludes the motivation, theory, implementation, exposition, and analysis of the Fast Monte Carlo simulation for the Linear Collider Detector, written by Daniel Furse during the summer of 2005 on the Department of Energy SULI summer internship at the Stanford Linear Accelerator Center. For any additional questions or comments, please contact Daniel Furse via email at:

<gtg251g@mail.gatech.edu>

\section{Acknowledgements}

First of all, the author would like to thank the Department of Energy, by whose dedication to scientific education this experience was made possible. The author would also like to warmly thank the following persons:

\begin{tabular}{lr}
\hline James Lindesay & Director of the SULI program at SLAC \\
Timothy Barklow & The author's mentor at SLAC \\
Norman Graf & for his extensive help and guidance \\
Helen Quinn & Official SULI program Contact \\
Pauline Wethington & SULI administrator \\
\hline
\end{tabular}

The author would finally like to extend his deepest gratitude to the other SULI interns and SLAC employees for making his summer research experience so unbelievably stimulating and truly unforgettable. 